Article

\title{
Day-Ahead Prediction of Microgrid Electricity Demand Using a Hybrid Artificial Intelligence Model
}

\author{
Yuan-Jia Ma and Ming-Yue Zhai * \\ School of Electronic Information Engineering, Guangdong University of Petrochemical Technology, No. 139 \\ Guandu Road, Maoming 525000, China; yuanjia.ma@gdupt.edu.cn \\ * Correspondence: caizhai@gdupt.edu.cn; Tel.: +86-188-1139-9766
}

Received: 21 April 2019; Accepted: 23 May 2019; Published: 30 May 2019

\begin{abstract}
Improved-performance day-ahead electricity demand forecast is important to deliver necessary information for right decision of energy management of microgrids. It supports microgrid operators and stakeholders to have better decisions on microgrid flexibility, stability and control. The available conventional forecasting methods for electricity demand at national or regional level are not effective for electricity demand forecasting in microgrids. This is due to the fact that the electricity consumption in microgrids is many times less than the regional or national demands and it is highly volatile. In this paper, an integrated Artificial Intelligence (AI) based approach consisting of Wavelet Transform (WT), Simulated Annealing (SA) and Feedforward Artificial Neural Network (FFANN) is devised for day-ahead prediction of electric power consumption in microgrids. The FFANN is the basic forecasting engine of the proposed model. The WT is utilized to extract relevant features of the target variable (electric load data series) to obtain a cluster of enhanced-feature subseries. The extracted subseries of the past values of the electric load demand data are employed as the target variables to model the FFANN. The SA optimization technique is employed to obtain the optimal values of the FFANN weight parameters during the training process. Historical information of actual electricity consumption, meteorological variables, daily variations, weekly variations, and working/non-working day indicators have been employed to develop the forecasting tool of the devised integrated AI based approach. The approach is validated using electricity demand data of an operational microgrid in Beijing, China. The prediction results are presented for future testing days with one-hour time interval. The validation results demonstrated that the devised approach is capable to forecast the microgrid electricity demand with acceptably small error and reasonably short computation time. Moreover, the prediction performance of the devised approach has been evaluated relative to other four approaches and resulted in better prediction accuracy.
\end{abstract}

Keywords: artificial intelligence; feedforward artificial neural network; simulated annealing; wavelet transform; electricity demand; forecasting; microgrid; smart grid

\section{Introduction}

In the conventional electric power system energy is produced by huge generation plants installed far away from load points. This causes transmission power loss and obstructs the opportunity of localizing power production, causing a much reliance on huge power generation systems. Recently, a global conceptual energy shift has been devised to make the existing power generation system more reliable and smart, in order to address the challenges the current power grid is facing [1,2].

According to these new ideas and to improve reliability and optimize energy usage, electric utilities and other stakeholders are continuously managing to adjust power generation to the consumption. 
Considering the fact that it is very hard to accumulate energy at a huge amount, energy supply shall be calibrated to match the consumption immediately. In view of that, it is significant that electricity demand prediction shall achieve smaller error or higher accuracy.

However, electricity demand relies on several facts, as the weather parameters (temperature, humidity, wind speed, cloud, rain, etc.), the daylight duration, current time, day, month, season, workability (presence of work) condition, etc. This makes electricity demand prediction rather a highly sophisticated task that looks beyond merely statistical tools. Recently, electric load prediction is being carried out employing many forecasting algorithms, and among them, ANNs are one of the most prominent choices due to their capability to acquire knowledge from experience (environment) [3].

In the modern power system era, the requirement for realizing the electricity supply vs. demand balance has contributed to the advent of small-scale electricity production and consumption atmospheres known as microgrids. In microgrids, customization of supply to demand can be carried out much more automatically because of their dispersed small-size components and the spatial closeness of all components (that further assists minimize transmission loses and costs).

The load curve in a microgrid and other small regions is different from typical electric power consumption curve, making conventional techniques (developed for national or regional prediction) inappropriate for its straightforward application due to two bold reasons. In microgrids and small regions (homes, buildings, local prosumers), not only the total electricity demand level is many times less than in the regional or national demand levels, but also the electricity demand profile manifests a more fluctuation and doesn't generally follow same profile.

A microgrid is a cluster of coupled loads and distributed generations (micro-gas-turbine, fuel-cell, combustion engine generator, energy storages, renewables, and etc.). Microgrids are at distribution voltage levels and have clear electrical boundaries [4]. They can operate either in islanded (isolated from the main electric power grid) or grid-connected mode (in parallel with the main grid) [5]. Thus, they have the latency to turn out to be independent. That means they are autonomous energy systems while they may stay coupled to the larger power grid to permit energy exchanges (sending or receiving) with the upper utility grid. The integration of Information and Communications Technologies (ICTs) assisted intelligent operation, control, supervision and protection functions into microgrids creates smart grids of desired sizes. In this scenario, to properly manage the electricity generation of its energy production resources, independent electricity demand forecasting is required for each particular microgrid in question.

The recent development and deployment of smart load (power) meters (SLM) at load points will allow utilities to correctly distinguish their electricity consumption behaviors. The operator of microgrid will collect further accurate values from distributed load curves that will allow the operator, for instance, to execute further accurate demand management and perform further reliable microgrid total load demand predictions employing the distributed data.

Electric load demand prediction is a rigorous task, as there are several dominant pertinent variables that should be taken into account, and a number of approaches have been employed to handle this sophisticated process. Prediction approaches can be categorized based on the facts taken into account as time-series and regression models. The time-series approaches describe future electricity demand based on its previous and present time series features [6-10]. The regression (causal) approaches characterize electricity demand based on external (natural or social) features that can possibly affect the electricity consumption [11-18].

$\mathrm{AI}$ and machine/deep learning based approaches have been used for electricity demand predictions. For instance, expert systems $[19,20]$, fuzzy logic systems [21] and neuro-fuzzy systems [22,23]. The expert system method designed [19] a methodological interval type-2 fuzzy logic system using extreme learning machine for electricity demand prediction. Expert system based AI methods have been reviewed for electricity demand prediction problem in smart grids [20]. Fuzzy logic based approach is proposed for short-term electric load forecasting using factorized weather parameters [21]. Neuro-fuzzy time series regression method is devised for electric load prediction [22]. 
The hybrid of wavelet transform and ANFIS is proposed for short-term electricity demand forecasting [23].

However, most of the abovementioned methods were developed for large-scale load demand levels such as national or regional levels, which have different characteristics from the electric load demand in microgrids. These methods are not effective for microgrid electricity demand data since the microgrid electric load demand data is much smaller and highly fluctuating compared to national or regional electric consumption data. Hence, other systematic method is needed for microgrid electric load demand data forecasting as proposed in this paper. Moreover, most of the previous methods did not combine the wind generation actual power output and external predictor variables such as weather parameters for a possible accuracy improvement that could be achieved.

It is observed that, the AI and machine/deep learning based electricity demand forecasting methods have given better forecasting accuracy than the conventional methods (such as persistence, linear regression, nonlinear regression, ARIMA, etc.).

Artificial neural networks (ANNs), in different forms, among the famous AI methods have been widely utilized for electricity demand prediction. For instance, Multi-layer Perceptron (MLP) ANN [24], RBF ANN [25], SOM ANN [26], and feedforward multi-layer (FFML) ANN [27].

$\mathrm{AI}$ and machine learning techniques have also been proposed and implemented for energy production forecasting in microgrids. For example, Physic Hybrid Artificial Neural Network (PHANN) model is proposed [28] for photovoltaic (PV) hourly day-ahead power forecasting in a microgrid framework. Different training approaches were compared for PV power production prediction based on machine learning tools [29]. The application of computational intelligence methods for day-ahead solar power forecasting has also been investigated and implemented using three different machine learning approaches [30].

In the recent few years, AI based approaches consisting of ANNs have become the most prominent techniques for electric load demand forecasting, with better performances than the others do.

The review in this paper shows that most of the electricity demand forecasting models have been developed to forecast electric load demands at national or regional levels. However, there have been only very few studies on electricity demand forecasting at small scale levels, such as microgrid, residential entity, commercial area, industrial unit, single building or household.

This study takes into account several predictor variables such as aggregated microgrid electric load data and meteorological variables for improved forecasting performance and accuracy.

Besides, most of the prior Feedforward Artificial Neural Network (FFANN) based load demand forecasting approaches have used a Back Propagation (BP) learning method to obtain the FFANN weight parameters. The BP algorithm can allow the FFANN to learn and model the electric load demand pattern. While running to find the values of the weight parameters during the training process, the BP performs a gradient descent inside the solution space in the direction of the global least value. Although BP training technique needs less computation time, it may be trapped by local solution and are therefore not capable to reach global solution.

To recompense the problems of the BP for FFANN training, this study uses the SA optimization algorithm.

Unlike the previous researches on FFANN based load demand forecasting, the current study employs a wavelet transform (WT) technique as a feature extraction technique to pick the most relevant features of the predictor variables for the SA-FFANN model.

Moreover, as far as we have reviewed, the WT-SA-FFANN based integrated approach has never been used for electric load forecasting.

In this study, a new and effective AI-based day-ahead microgrid electricity demand forecasting approach using the integration of FFANN, SA and WT is devised. The devised WT-SA-FFANN based integrated electricity demand forecasting approach is compared with Persistence, BP-FFANN (back-propagation feedforward ANN), Genetic Algorithm (GA)-FFANN (GA combined with FFANN), 
and Particle Swarm Optimization (PSO)-FFANN (PSO combined with FFANN), to demonstrate its robustness regarding prediction accuracy and other performance indexes.

The main contributions of this study are:

(1) Provide a new and effective AI-based hybrid method for day-ahead electric load prediction in microgrids considering actual electricity demand, meteorological variables and other derivative data and input factors;

(2) Improve electricity demand forecasting accuracy;

(3) Deliver a hands-on solution to electricity demand prediction problems in microgrids and other small-scale energy systems.

The study is organized as follows. Section 2 presents the devised prediction approach and model framework. The various performance criteria used to estimate the electricity demand prediction accuracy are given in Section 3. Section 4 describes the case study, experimental results and discussions. The study is finally summarized in Section 5 .

\section{Proposed 24 h-Ahead Electricity Demand Prediction Strategy}

Electric load forecasting plays a key role for short-term operational planning and long-term design planning of microgrids. Prediction is one of the main functions of the energy management system (EMS) that is recently being developed for operational control of microgrids.

Day-ahead or short-term electricity demand prediction is an important aspect of the energy resource management such as generation control, economic dispatch, energy management optimization, demand response, and security analysis of microgrids. Conventionally, load forecast analyses were carried out for big entities such as regions or nations. Yet, with the evolution of the idea of smart-grids, the research of the electricity demand characteristics and forecast of smaller environments, such as microgrids, has become a major focus area to assist an advanced management of the power network.

This study proposes a new microgrid electricity demand prediction model using the hybridization of WT, SA and FFANN. The WT is utilized to extract relevant features of the target variable (electric load data series) to obtain a cluster of enhanced-feature subseries. The extracted subseries of the past values of the electric load demand data are employed as the target variables to model the FFANN. While the predictor set containing historical lag values of electricity demand, weather variables and seasonal (hourly, daily, weekly, monthly, and seasonally) variations are used as input variables for the FFANN modeling. Hence, these predictor variables as training inputs and the electricity demand WT subseries as training variables are used to train the FFANN forecasting engine in this work.

The future values of the predictor values are then employed to predict the next electricity demand subseries based on the trained FFANN model. The SA optimization searches for the best parameter set of the FFANN weights to attain improved prediction accuracy. Lastly, the predicted or next (ahead) electricity demand time series is reassembled by employing an inverse WT on the predicted subseries.

A one-year training window (from May 2014 to April 2015) information of historical electric load data, weather data (temperature and humidity), daily variations (hours in days), weekly variations (days in weeks), and workability conditions (weekday or weekend/holiday) has been used to construct the forecast model. The model is validated with actual electricity demand information from an operating industrial microgrid in Beijing, China. The effectiveness of the devised electricity demand forecast model is tested with a one year (from May 2015 to April 2016) testing window information. The forecast results are presented for future days ( $24 \mathrm{~h}$ ahead formats) with one-hour time interval base. The forecast model of the devised AI-based integrated strategy has the ability to relearn any time when there is new learning dataset. The devised electricity demand forecasting schematic is shown in Figure 1. 


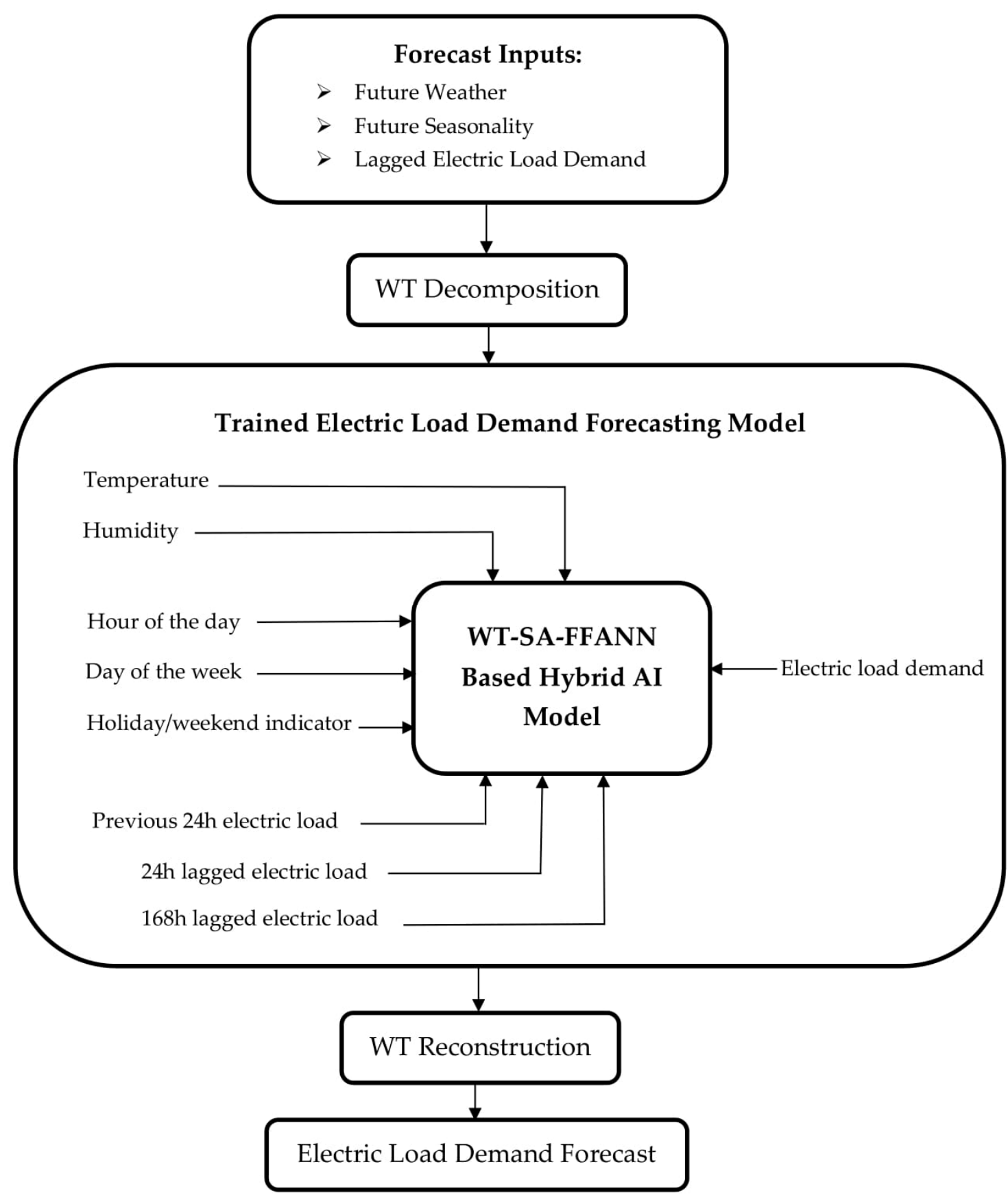

Figure 1. Schematic for the devised WT-SA-FFANN based integrated electricity demand forecasting approach for microgrids.

The detail operation and calibration of the integrated AI-based approach employed to implement the devised electric load prediction approach is described stepwise in Table 1.

As it is clearly depicted in Algorithm 1, the WT tools are used in the first and final steps of the forecasting process. The historical target (electricity demand) data series is first decomposed into several subseries by the WT. The decomposed historical subseries electricity demand as the training target and the predictors set as the training inputs are used to train the FFANN model using the SA optimization method. The trained FFANN model maps the nonlinear relationship between the predictor variables and electric load. Then, the WT decomposed subseries data of future (next day) predictors are fed to the developed (trained) SA-FFANN model to predict the future electricity demand 
subseries of the microgrid. Lastly, the future electricity demand subseries are reassembled to give the desired electricity demand forecast.

Algorithm 1 Algorithm of the proposed hybrid WT-SA-FFANN based electricity demand forecasting approach

1. Begin

2. Enter historical dataset (raw data)

2.1. Historical microgrid electricity demand data

2.2. Historical weather data

2.3. Historical seasonal variations

3. Apply data treatment

3.1. Change all data to hourly resolution via averaging

3.2. Synchronize the time zones of all the data sources

3.3. Fill skipped data points, if any

4. Apply the WT decomposition to the historical target dataset (electricity demand)

5. Identify training datasets

5.1. Training dataset: Historical predictor dataset versus electrciry demand subseries dataset

6. Initialize FFANN model

7. Set initial parameters of weights \& biases for the intialized FFANN model

8. Train the initialized FFANN model with the Training dataset using the SA parameter optimization

9. Check convergence of FFANN model

9.1. Save the trained model if the convergence condition is met (this saved model is called Electricty Demand Forecaster)

9.2. Otherwise, go to step (7)

10. Enter future predictor dataset

11. Apply data treatment

11.1. Change all data to hourly resolution via averaging

11.2. Synchronize the time zones of all the data sources

11.3. Fill skipped data points, if any

12. Input the processed future predictor dataset to the Electricity Demand Forecaster saved in step (9.1), and obtain the electricity demand forecast subseries of the microgrid

13. Apply inverse WT on the electricity demand forecast subseries obtained and obtain the electricity demand forecast data for the desired time ahead (day-ahead in this paper)

14. End.

The selected configuration type and parameters values of the FFANN model for the proposed electricity demand forecasting problem in this paper are given in Table 1. 
Table 1. Parameters of FFANN.

\begin{tabular}{ll}
\hline \multicolumn{1}{c}{ Parameter } & \multicolumn{1}{c}{ Value } \\
\hline Configuration & Feedforward with 3 layers \\
Hidden layer size & 1 \\
Hidden neuron quantity & 20 \\
Hidden layer neurons activation & Tangent sigmoid (tansig) \\
Output layer size & 1 \\
Output neuron quantity & 1 \\
Output layer neurons activation & Pure linear (purelin) \\
Training ratio & 0.01 \\
Epochs & 1000 \\
Training target error & 0.001 \\
\hline
\end{tabular}

\section{Forecasting Accuracy Assessment}

To measure the accuracy of the proposed hybrid AI-based WT-SA-FFANN electric load prediction method, the MAPE, RMSE, NMAE, SDE, and FS criteria are used.

The MAPE (mean-absolute-percent-error) is given as [31,32]:

$$
M A P E=\frac{100}{N} \sum_{h=1}^{N}\left|\frac{P_{h}^{a}-P_{h}^{f}}{P_{h}^{a}}\right|
$$

where, $P_{h}^{a}$ and $P_{h}^{f}$ are the real and prediction values of the electricity demand at hour $h$, respectively, and $N$ is the forecasting horizon.

The RMSE (root-mean-squared-error) is defined as [31,32]:

$$
\text { RMSE }=\sqrt{\frac{1}{N} \sum_{h=1}^{N}\left(P_{h}^{a}-P_{h}^{f}\right)^{2}}
$$

The NMAE (normalized-mean-absolute-error) is given by [31,32]:

$$
\text { NMAE }=\frac{1}{N} \sum_{h=1}^{N} \frac{\left|P_{h}^{a}-P_{h}^{f}\right|}{P_{\text {peak }}}
$$

Here, $P_{\text {peak }}$ is the peak total load demand of the microgrid.

The SDE (standard deviation of error) is given by [32]:

$$
\begin{gathered}
\mathrm{SDE}=\frac{1}{N} \sum_{h=1}^{N}\left(e_{h}-\bar{e}\right) \\
e_{h}=P_{h}^{a}-P_{h}^{f} \\
\bar{e}=\frac{1}{N} \sum_{h=1}^{N} e_{h}
\end{gathered}
$$

where, $e_{h}$ is the prediction error at hour $\mathrm{h}$ and $\bar{e}$ is the average error in the prediction horizon.

The inconsistency of an electricity demand prediction approach, after development, is an index of the stochasticity of the approach, and is estimated by calculating the variance of the forecasting error. The prediction is considered to be highly accurate or certain if the result of the variance is lower [32]. 
Using Equation (1), daily error variance is given by [31,32]:

$$
\begin{gathered}
\sigma_{e, d a y}^{2}=\frac{1}{N} \sum_{h=1}^{N}\left(\left|\frac{P_{h}^{a}-P_{h}^{f}}{P_{h}^{a}}\right|-e_{\text {day }}\right)^{2} \\
e_{\text {day }}=\frac{1}{N} \sum_{h=1}^{N}\left|\frac{P_{h}^{a}-P_{h}^{f}}{P_{h}^{a}}\right|
\end{gathered}
$$

The FS (forecast-skill) metric calculates the worth of forecasting methods by referring the forecasting error obtained by methods to persistence forecast. For day-ahead forecast, the persistence output is defined as [31-33]:

$$
P_{h}^{f}(t)=P_{h}^{a}(t-24)
$$

The FS measure is estimated based on the relation of the RMSEs of prediction methods and persistence method [31,33]:

$$
\mathrm{FS}=1-\frac{\mathrm{RMSE}_{\text {Model }}}{\mathrm{RMSE}_{\text {Persistence }}}
$$

\section{Experimental Results and Discussions}

In this paper, the hybrid WT-SA-FFANN AI model is established for day-ahead electric load prediction in microgrids. Electricity demand data from a real microgrid energy system in Beijing, China is used to build and verify the prediction model. The microgrid system has a peak aggregate electricity demand of $2400 \mathrm{~kW}$.

\subsection{Data Sources and Treatment}

The dataset used to develop the devised electric load prediction model in this study are prior values of electric load data, weather data (temperature and humidity), daily variations (hours in days), weekly variations (days in weeks), and workability variations (weekday, weekend or holiday). The following subsections describe each of the data sources and types used to develop the proposed load forecasting model in this paper, and the data treatment techniques used as well.

\subsubsection{Electric Load Data}

The electric load data has taken from an actual (operation) microgrid energy system in Beijing, China. The SCADA in microgrids contains a comprehensive record of the various distributed load measurements collected from the load meters installed at different load points within the microgrid. The SCADA also presents the complete historical measurements of the aggregate electric load demand of the microgrid in ten-minutes and one-hour time intervals. In addition, the SCADA system presents a complete record of the status (operating or not at a specific time) of the various distributed loads of the microgrid and their associated power demands, which is very important for short-term electricity demand prediction model development. Hence, SCADA is a critical element of microgrids and plays a vital role in electricity demand forecasting. In this study, the electricity demand data used is a vector consisting of the past values of the microgrid aggregate electricity demand measurements for one year with 10-min resolution. That equals 52,560 measurement values of electricity demand.

A one year electricity demand curve of the microgrid is shown in Figure 2, with 10-min average values. 


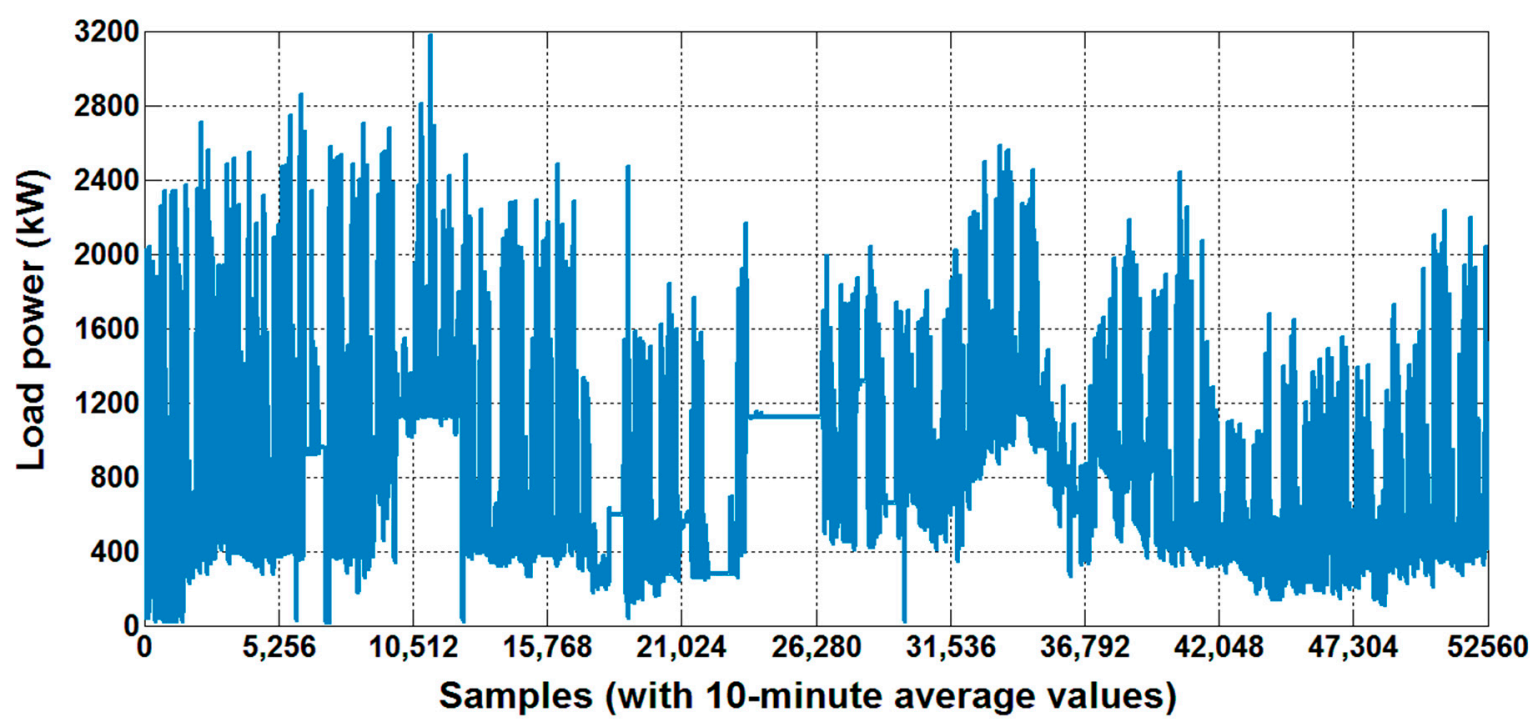

Figure 2. A one-year (1 May 2014-30 April 2015) load demand curve.

Sample daily load curves for weekdays and weekends/holidays, from all the seasons of the year, in the training dataset are also depicted, respectively, in Figures 3 and 4.
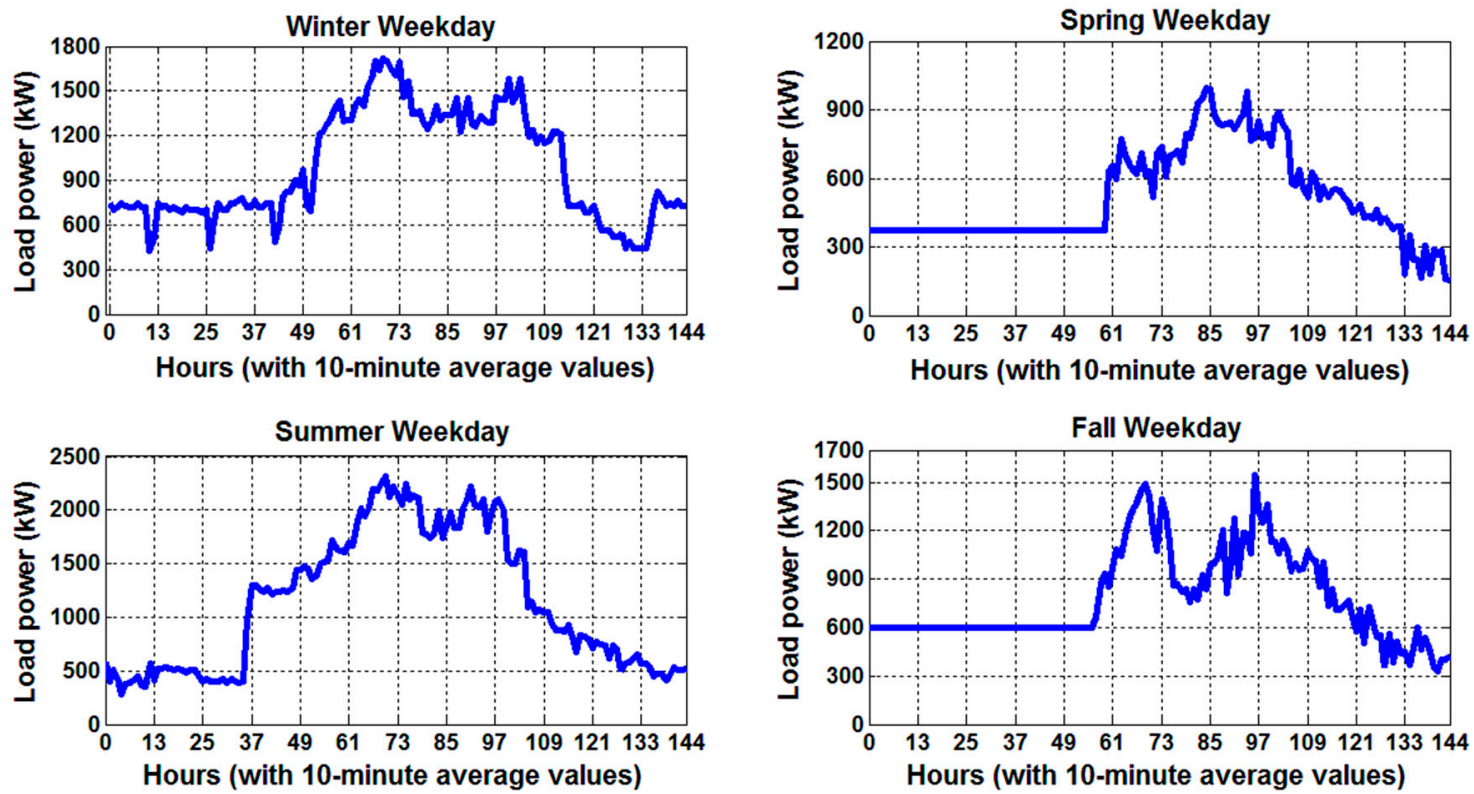

Figure 3. Sample weekday load demand curves, with 10-min average values; for winter weekday (Thursday, 15 January 2015), spring weekday (Wednesday, 8 April 2015), summer weekday (Thursday, 3 July 2014), and fall weekday (Friday, 10 October 2014). 

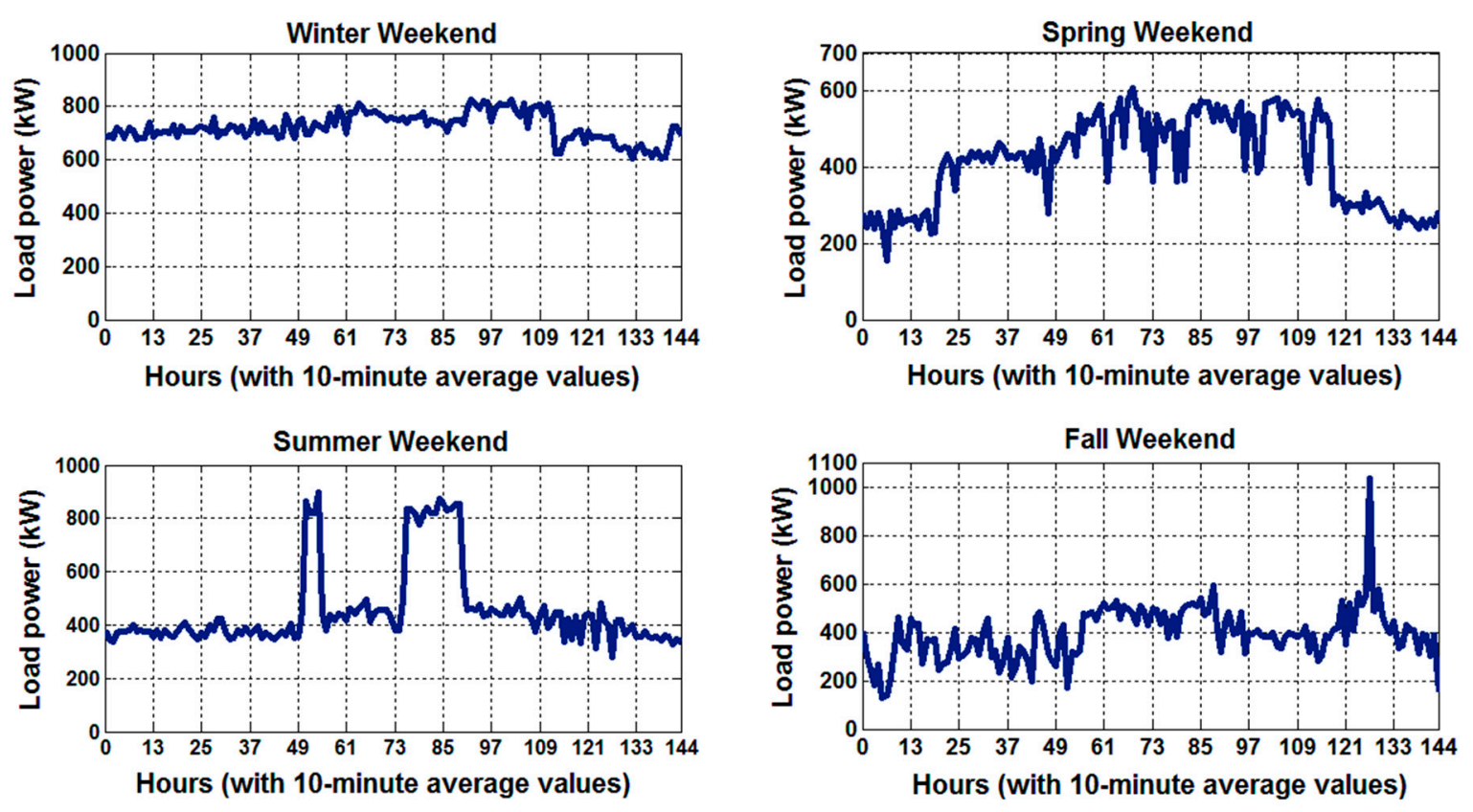

Figure 4. Sample weekend/holiday load demand curves, with 10-min average values; for winter weekend (Saturday, 3 January 2015), spring weekend (Sunday, 12 April 2015), summer weekend (Saturday, 5 July 2014), and fall weekend (Sunday, 12 October 2014).

As seen in these Figures 2-4, there is much difference regarding the electric load demand between weekdays (working days) and weekends/holidays (non-working days). Electricity consumption on weekends are considerably lower than that of weekdays in all the seasons. Unlike the load demands on weekdays, the load demand curves on weekends have less fluctuation throughout the days.

Moreover, the electricity consumptions in the winter and summer days are higher than that of the spring and fall days. This is due to higher demand of electricity for heating and cooling purposes, respectively, during winter and summer days.

The forecast model predictor variables related with the load data are derivatives of the historical load data, as the load demand profile shows some characteristic correlations with previous load profiles. For example, the previous $24 \mathrm{~h}$ average load data, $24 \mathrm{~h}$ lagged load data and $168 \mathrm{~h}$ lagged load data predictor variables are the mean electricity demand of the preceding day, the load at similar hour in the preceding day and the demand at similar hour in the preceding week, respectively.

\subsubsection{Weather Data}

The weather data (temperature and humidity) predictor variables have also a considerable impact on the load power forecasting, as the microgrid load consumption pattern exhibits some important characteristic distribution based on the external weather (environmental) conditions. In this paper, the electricity demand prediction model employs weather (temperature and humidity) forecasts close to the installation site of the microgrid. The weather data predictor variable is a matrix containing weather parameter values, organized in two columns. The columns correspond to the weather variables and holds the past values of weather parameters for one year with 15-min resolution. That equals 35,040 weather forecast values per column. Columns 1 represents air temperature forecast data and column 2 represents the humidity forecast data. In total, the weather data predictor variable matrix has 70,080 past values of meteorological forecasts.

The weather forecast is presented using the Greenwich Mean Time (GMT) scale that lags by $8 \mathrm{~h}$ from the Beijing time.

The 15-min average ambient temperature forecast by the weather research and forecast (WRF) model for one year is presented in Figure 5. 


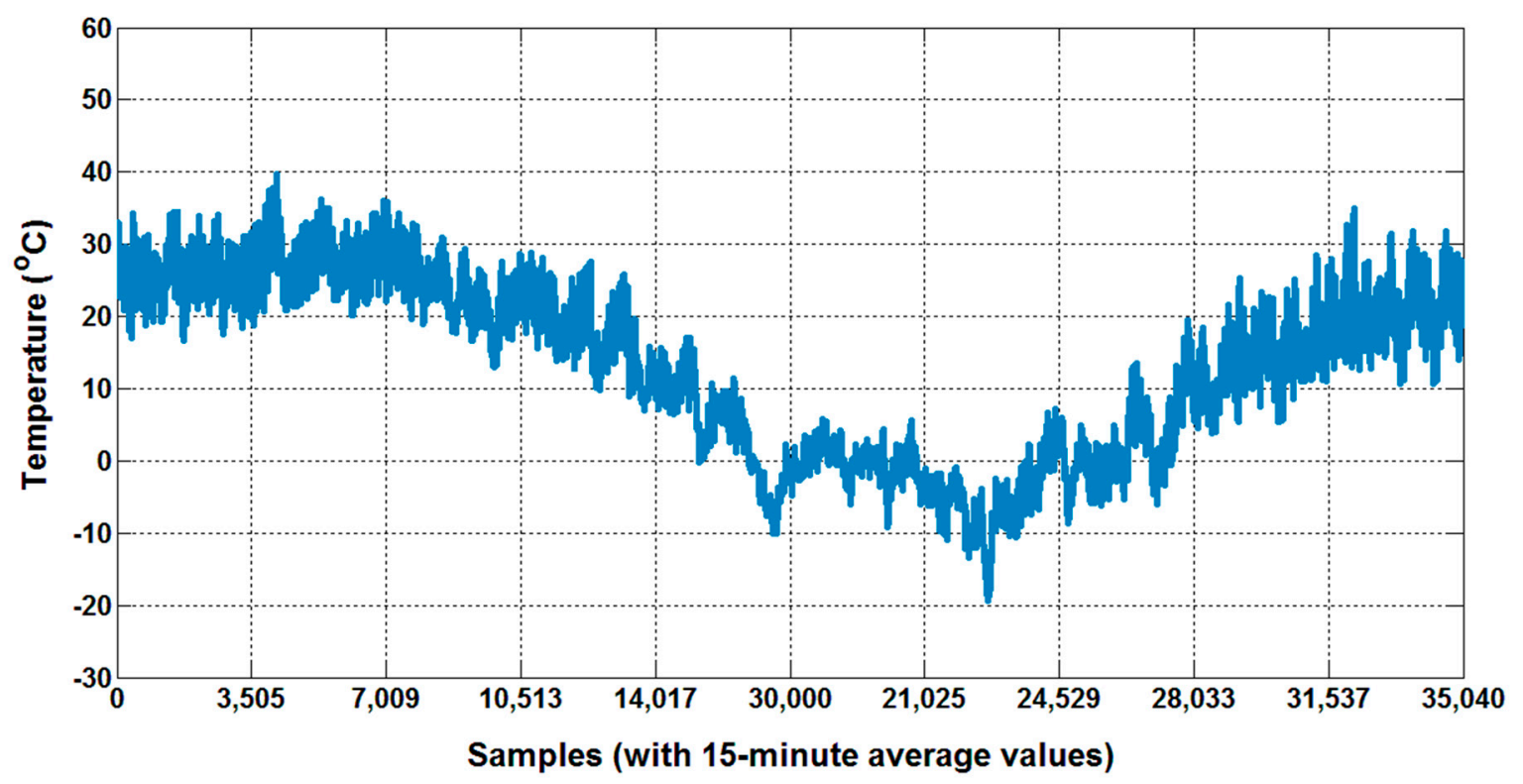

Figure 5. A one-year (1 May 2014-30 April 2015) WRF temperature forecast, with 15-min interval.

Similarly, the 15-min average air humidity forecast by the WRF model for one year is given in Figure 6.

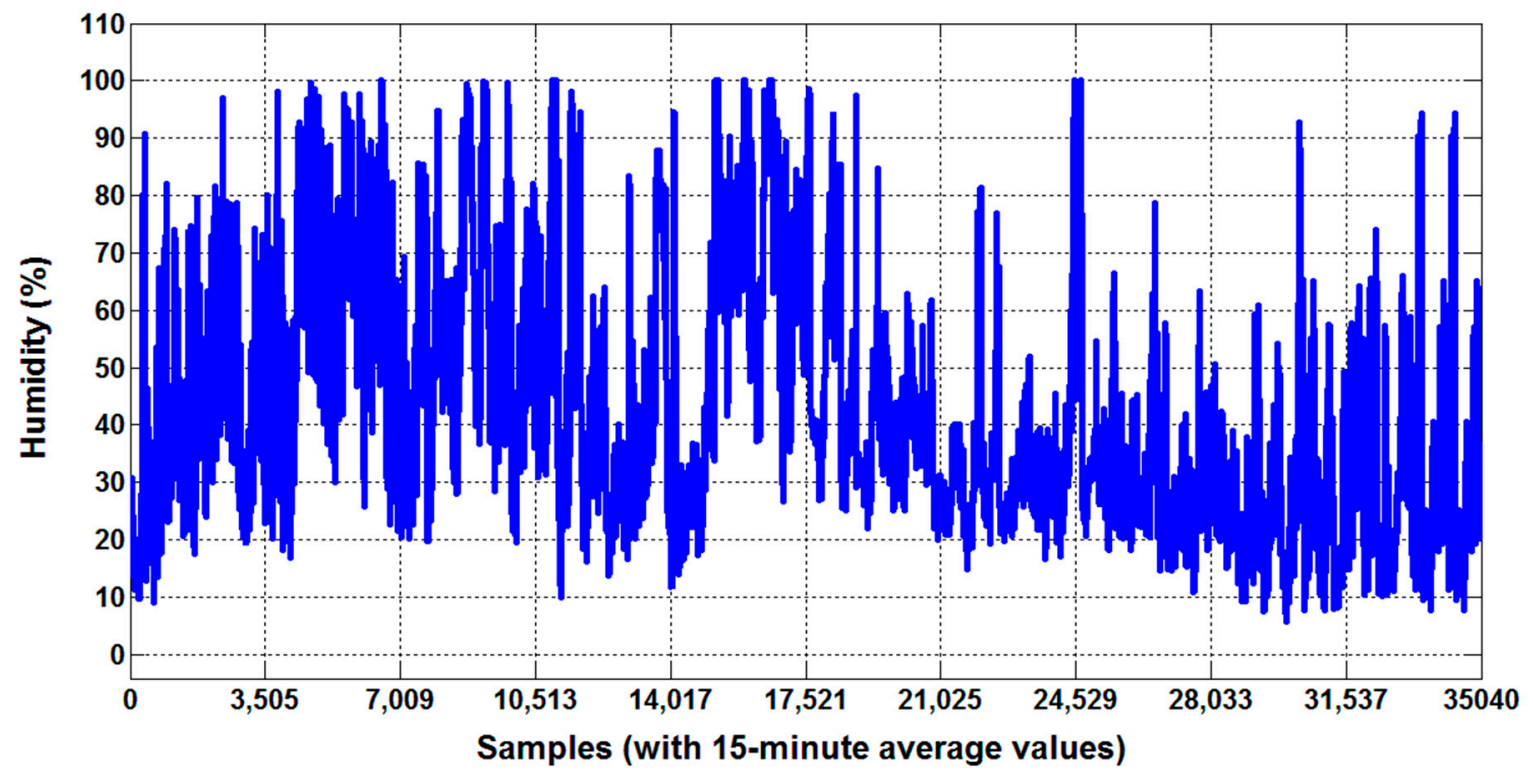

Figure 6. A one-year (1 May 2014-30 April 2015) WRF humidity forecast, with 15-min interval.

It can be seen that the seasonal effect of the air temperature and humidity is influential on the electricity demand of the microgrid by comparing Figure 2 with Figures 5 and 6 .

\subsubsection{Other Predictor Data Inputs—Natural/Social Sources}

The other important predictor variables used to construct the load forecast model are daily variation data, weekly variation data and workability condition data, as the electric load consumption pattern is also highly associated with these natural circumstances.

The daily variation data is a vector consisting of the hours of the day $(1,2, \ldots, 24)$ information for all the days of the model training window. 
As it can be observed in Figures 3 and 4, the microgrid electric load demands are different at each hour. Hence, hour in the days is considered as one of the predictor features as it may affect the load forecasting process.

The weekly variation data is a vector containing the days of the week $(1,2, \ldots 7)$ information for all the days in the model training window.

As it can be observed from Figures 3 and 4, the microgrid electric load demands slightly vary between weekdays and weekends. In fact, the load demand also varies among the days in the weekdays and/or weekends. Thus, day of the week is considered as one of the predictor variables as it may affect the forecasting process.

The workability condition data is a vector with numeric flag values $(0$ or 1$)$ for all the model-training days, a value of 0 indicates the specific day (hour) is a non-working day/holiday/weekend and 1 shows the working/weekday day. The list of non-working days (official holidays) for the devised electricity demand prediction model training and testing in the years 2014, 2015 and 2016 have been taken from the China Official National Holiday Schedule [34].

It is observed that the microgrid electric load demands at the holidays are lower than that of the non-holidays (working days). Hence, workability condition or indicator is considered as one of the predictor variables as it may affect the load prediction process.

\subsubsection{Data Preparation}

Some of the predictor variables should be processed to more simplified and matching representations ahead of the WT decomposition and FFANN learning.

The load power data and weather forecast data are changed into hourly (mean) data. The data is finally arranged in one-hour resolution to fit the resolution differences of the electricity demand and weather data sources.

The weather forecast data record time is converted to the Beijing time to synchronize it with the time-zone of the electricity demand record (microgrid SCADA time).

A single skipped data point is filled by the average of the data points at the same hour of the previous and future days, if any. However, timestamps with excess (more than one) skipped data points are not filled up by any data. These timestamps are removed from the forecast model training input dataset. This is because inserting excess additional (derived) data samples instead of the skipped actual data points may contaminate the training dataset and change the nature of the whole dataset.

\subsection{Proposed Framework for the AI-Based WT-SA-FFANN Integrated Forecasting Model}

\subsubsection{Wavelet Transform (WT)}

The amplitude of the electric load curve obtained from microgrid systems varies in each time instant as shown in Figures 2-4. Observation of the non-stationary and non-linear load curves of microgrids calls for the decomposition of the original electric load data time series into various subseries elements, to extract the utmost significant characteristics of the data. The WT tool is employed to decompose the target variable (microgrid electricity consumption) data series to a cluster of data subseries. The resultant decomposed data subseries can provide improved performance features than the original data. Thus, in this work, the decomposed (subseries) data is utilized to construct the electricity demand model to achieve less prediction error.

The rational for the effectiveness of the decomposed data subseries for the prediction is due to the enhanced data decomposition (extraction) capability of the WT algorithm.

WT decomposes the forecast model target variable dataset into a group of subseries. It extracts new set of features from the original target variable. The resulting new features provide enhanced performance than the original target feature. Therefore, using the new features derived from the WT decomposition instead of the original target variable (electricity demand) will definitely enhance the 
performance of the electricity demand forecast. The main reason for the enhanced performance of the new data features obtained from the WT decomposition is due to the data filtering capability of the WT.

There are two types of wavelet transform. They are continuous wavelet transform (C-WT) and discrete wavelet transform (D-WT).

The C-WT WT $(p, q)$ of a signal $h(x)$ with reference to a mother wavelet $\Phi(x)$ is formulated below [35].

$$
W T(p, q)=\frac{1}{\sqrt{p}} \int_{-\infty}^{+\infty} h(x) . \Phi\left(\frac{x-q}{p}\right) d x
$$

Here, $p$ is the wavelet scaling factor that controls the scattering of the wavelet and $q$ is the wavelet transformation factor that determines the center of the wavelet. The D-WT $W T(r, s)$ is as precise as the C-WT but further efficiently powerful [36]. D-WT is formulated as follows.

$$
W T(r, s)=2^{-(r / 2)} \sum_{t=0}^{T-1} h(t) . \Phi\left(\frac{t-s 2^{r}}{2^{r}}\right)
$$

Here, $T$ is the size of the signal $h(t)$. The scaling factor $p$ and transformation factor $q$ are functions of the integer parameters $r$ and $s$ (i.e., $p=2^{r}, q=s 2^{r}$ ), and $\mathrm{t}$ is the discrete time.

Mallat et al. [37] developed a very quick and efficient D-WT algorithm relying on the four fundamental filter types, namely low pass decomposition, high pass decomposition, low pass reconstruction, and high pass reconstruction filters. Multi-resolution using the Mallat decomposition and reconstruction techniques is a way to obtain "approximation" and "detail" components of a particular signal. Through sequential decomposition of the approximation, a multistep ranked decomposition is achieved. That means the initial given signal is decomposed to reduced resolution sub-signals.

In this paper, a Daubechies order $4(\mathrm{Db} 4)$ wavelet function is employed as the mother wavelet $\phi(t)$. Db4 $\phi(t)$ gives an acceptable trade-off between evenness and wavelength, providing a relevant feature for short-term electricity demand forecasting.

Wavelet transformations have been applied by prior research groups for various applications. For example, wavelet transforms are employed for a hybrid electricity demand prediction $[35,36]$, electricity market price prediction [38] and solar power forecasting [31].

Figure 7 shows the original electricity demand data illustrated in Figure 2 and the WT extracted features. 

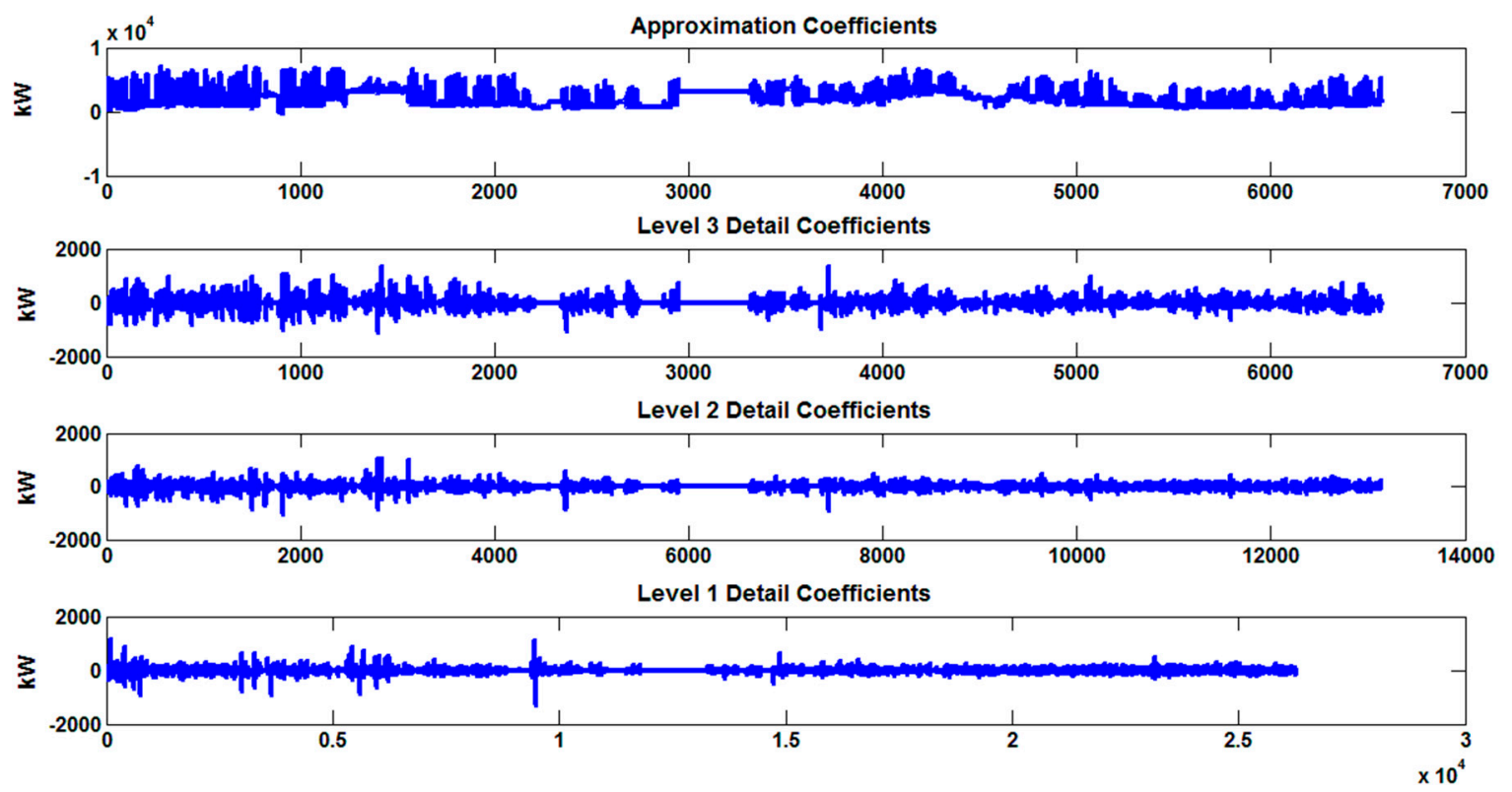

Figure 7. Original electricity demand data and its WT extracted features.

\subsubsection{Simulated Annealing (SA) Optimization}

Simulated Annealing (SA) is a probabilistic method for obtaining the global best solution of a given optimization problem. Precisely, it is an Artificial Intelligence (AI) based metaheuristic technique to find a global optimal solution in a broad search domain for a function. SA is a better approach than gradient descent based methods when obtaining a global optimal solution is more significant than obtaining a specific local optimal solution in a given time.

The term and motivation originate from annealing in metallurgy, a method in which melting and regulated condensing of a metal to raise the dimension of its constitutes and decrease their imperfections. The target of this annealing process is to achieve the minimum energy state. Melting and condensation are features of a metal that rely on its thermal characteristics. Melting and condensing the metal influences both the temperature and thermal free energy. The SA algorithm can be employed to search a global minimum for an optimization problem containing several decision attributes $[39,40]$. The concept of gradual condensation employed in the SA algorithm is considered as a gradual reduction in the probability of obtaining bad solutions while the solution domain is discovered. Obtaining bad solutions is an essential behavior of metaheuristics since it enables for an additional broad search for the global best solution.

To improve the forecasting performance of electricity demand prediction models, the SA algorithm is applied to search the optimal parameter set of the models. Specifically in this paper, the SA algorithm is employed to find the optimal parameters (neuron connection weights) of the FFANN electricity demand forecasting model.

Reference [41] devised an algorithm to model the annealing process, simulating the change of a material from a higher temperature to thermal stability. The correlation between the gradual condensation of a material and minimizing an optimization problem was first recommended [42].

The brief operating mechanism and implementation of the SA optimization algorithm used for training the FFANN electricity demand forecasting model is presented next.

In order to minimize the function $\mathrm{f}(X)$, a number of initial parameters of the SA algorithm should be set. These parameters are initial temperature $T_{0}$, initial solution weight vector $X$ and solution weight step length vector $V$. $X$ and $V$ are vectors of dimension $n$ that equals with the number of the FFANN weights. $x$ and $v$ are members of $X$ and $V$ respectively. We express $f(X)$ as the sum of squared error quantity that is generated by $X$. Using the starting values of $X$, the weights are employed for the FFANN to compute and record its $f$ value as the present best optimal value. A candidate solution $Y$ is 
then selected by updating every $x_{i}$ through Equation (13), where $\mathrm{rad}$ is an arbitrary number taken from a normal distribution in the range $[-1,1]$.

$$
y_{i}=x_{i}+r a d * v_{i}
$$

The functional value $f \prime$ is then calculated using the new candidate solution $Y$. If $f \prime$ is smaller than $f$, then the $Y$ is taken as the new solution and $X$ is replaced by $Y$. If the present $f$ is still the minimum solution, then $f$ and the associated $X$ are recorded as the optimal solution. If $f \prime$ is larger than or equal to $f$, then the Metropolis benchmark is applied to check the recognition of the solution. The value of the equation expressed in Equation (14) is calculated and crosschecked with $p \prime$, an arbitrary number taken from a normal distribution in the range [0,1]. If $p$ is larger than $p^{\prime}$, the new candidate solution is recognized and $X$ is replaced by $Y$, else, $Y$ is refused.

$$
p=e^{\left(\frac{f(x)-f(y)}{T}\right)}
$$

The process iterates for a specified number of iterations $N_{I}$ for all members of $X$. The value of $N_{I}$ used in this paper is given in Table 1. In the iterations, the step length $V$ is updated in such a way that $50 \%$ of all the movements are taken. $T$ is minimized after $N_{T}$ epochs of the abovementioned procedure. Investigation of Equation (14), demonstrations a reduction in $T$ reduces the probability of taking uphill movements. The decrease in $T$ is computed using Equation (15). $r_{T}$ is a specified value in the range $[0,1]$.

$$
T_{1}=r_{T} * T_{0}
$$

The first updated candidate solution tested after a decrease in temperature $(T)$ comes to be the present optimal solution. A reduction in temperature brings the number of refusals to rise and the step lengths to decrease. Convergence of the SA algorithm happens when the functional value for the final temperature $(T)$ decrease varies from the associated value at the present temperature by smaller than the error threshold $N_{\varepsilon}$ and the last functional value at the present $T$ varies from the present optimum function by smaller than $N_{\varepsilon}$. The SA algorithm escapes from premature convergence by recognizing uphill movements. When the algorithm runs and the decrease in temperature happens, recognition of uphill movements and step lengths reduce, and hence global optimal solution is achieved.

The values of the parameters used for the SA algorithm are provided in Table 2.

Table 2. Parameters of the SA algorithm.

\begin{tabular}{ll}
\hline \multicolumn{1}{c}{ Parameter } & \multicolumn{1}{c}{ Value } \\
\hline Number of iterations & 1000 \\
Size of solution vector & Number of FFANN neuron connection weights \\
Initial temperature & 200 \\
Temperature update function & Exponential temperature update \\
Temperature reduction factor & 0.85 \\
Max. number of inner loops & 100 \\
Max. number of movements in inner loops & 50 \\
Max. number of failed inner loops & 3 \\
Cooling rate & 0.95 \\
\hline
\end{tabular}

Figure 8 shows the flowchart of the SA algorithm. 


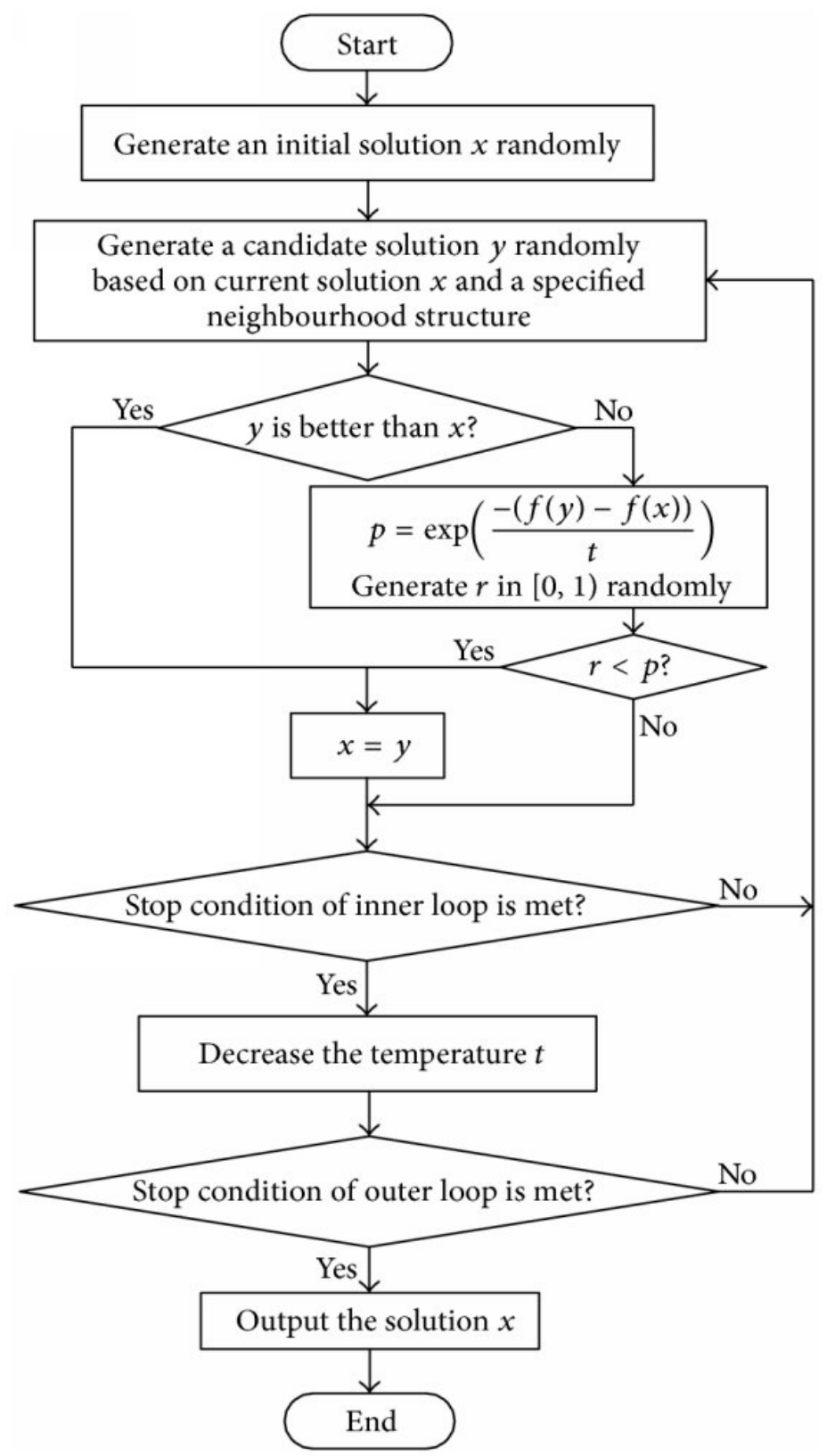

Figure 8. Flowchart of the SA algorithm.

\subsubsection{Feedforward Artificial Neural Network (FFANN)}

Artificial neural network (ANN) is a robust data processing (regression or classification) model, which is able to capture an existing complex relationship within a dataset. ANN is able to learn fast the behavior of the data and acquire knowledge. The development of the ANN model was motivated by the method the human biological nervous systems, specially the brain, process information. The important characteristics of the ANN model is its unique configuration for information manipulation. It is consists of plenty of information manipulating constituents known as neurons configured in various hierarchical layers. The layers are input, hidden and output layers. The neurons making up the input, hidden, output layers are respectively called input, hidden and output neurons. These neurons are connected through certain scaled connections.

The neuron connecting scales are known as weights. The neurons in ANN operate in combination to solve a given real-world problem (fitting, approximation, regression, pattern recognition, classification, etc.) [32]. 
There are different types of ANNs based on the configuration (connection arrangement) of the neurons and the flow of information. ANNs can be categorized as feedforward artificial neural network (static network) and recurrent artificial neural network (dynamic network). The FFANNs have no feedback components and hold no delays. In FFANN, information flows right from inputs to output in the forward direction. The model output is computed right from the input employing the feedforward connection weights. While in the recurrent neural network models, the output relies not only on the present inputs to the model, but also on the present or prior inputs, outputs, or states of the model. Although they are effective for high dimension and very complex problems, the recurrent neural networks are complex for implementation and computationally exhaustive. On the other hand, FFANNs are easy to implement, fast and very effective for reduced-dimension data processing problems such as forecasting.

While developing a FFANN model for solving a specific problem, the quantity of the hidden layer neurons should be chosen properly with a great care. Nevertheless, there exist no clear technique for optimal sizing of the number of neurons at the hidden layer. In this study, we determine the number of hidden layer neurons through extensive and continuous experimentation, which is called empirical parameterization process. Various configurations of FFANN with various number of neurons at the hidden layer were examined. The best FFANN model configuration was selected using the root mean squared error (RMSE) measure between the model output value and actual target value.

The chosen FFANN model configuration for the proposed electricity demand forecasting task in this paper is shown schematically in Figure 9.

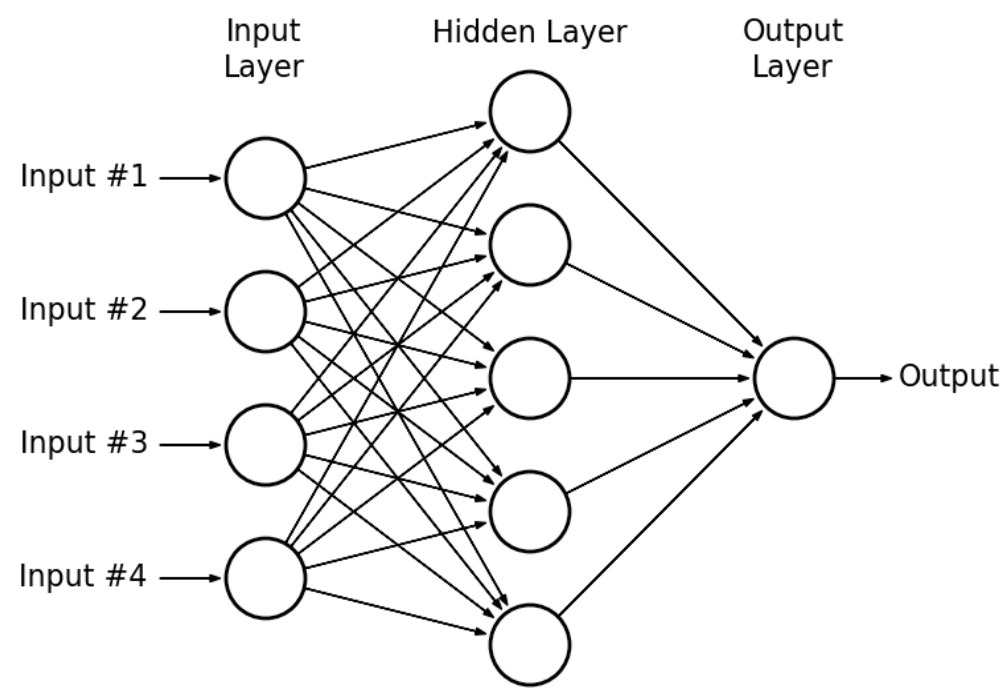

Figure 9. Structure of FFANN.

The mathematical modeling depiction of the ith neuron in the FFANN model is illustrated in Figure 10 [32].

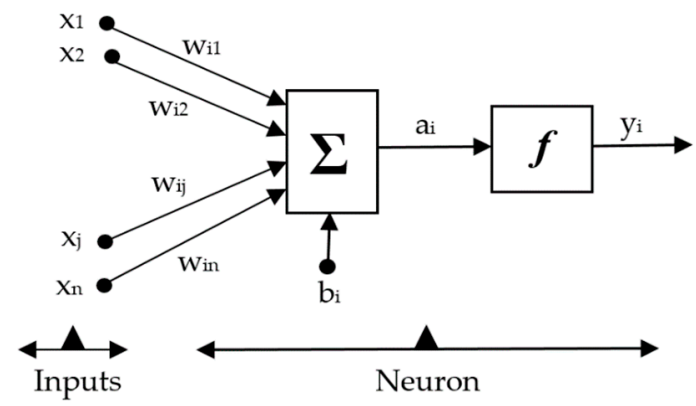

Figure 10. Math model of a neuron of FFANN. 
In Figure 10, the $x$ is the input to the $i$ th neuron, $y_{i}$ is output, $w_{i j}$ is a connecting weight; $b_{i}$ is a bias quantity (usually constant), and $f_{i}$ is known as the activation function. The activation function carries out a major task during the FFANN model training. It controls the behavior of the neurons' output. Based on Figure 10, the FFANN neuron output can be formulated as follows:

$$
y_{i}=f_{i}\left(\sum_{j=1}^{n} w_{i j} \cdot x_{j}+b_{i}\right)
$$

Normally, FFANN is developed using the Back Propagation (BP) learning algorithm. In the BP learning of the FFANN, the neuron connecting weights are adjusted employing the BP algorithm over the given input-output dataset. This greatly helps the FFANN model to learn the behavior of the data very fast. While running to find the values of the FFANN weight parameters during the training process, the BP performs a gradient descent inside the solution space in the direction of the global least value. Although BP training technique needs less computation time, it may be stuck by suboptimal (local) solution and are therefore not capable to reach global (system-level) optimal solution. Hence, the BP training of the FFANN model does not guarantee reliable electricity demand forecasting accuracy throughout the entire forecasting horizon and scenario.

To compensate the drawbacks of the BP and improved-performance FFANN training, this paper uses the SA algorithm, which is capable to obtain global optimal solutions with faster convergence rate and computation time.

Hence, the SA training of the FFANN model will result global optimal values for the FFANN connection weights, which corresponds to a higher electricity demand forecasting accuracy.

The SA optimization technique is computationally simple and fast convergent for a given configuration of the FFANN model.

In this work, the FFANN model weights are implemented as parameters of the SA. The mean squared error (MSE) between the model output and actual target is formulated as the cost (fitness) function of the SA algorithm. The goal of devised strategy is to obtain the smallest value of this fitness function. This procedure iterates until the prediction error achieves the intended level.

\subsection{Forecasting Results and Statistical Analysis}

As abovementioned, the input variables used for constructing the forecast model are ambient temperature, air humidity, hours in the days, days in the weeks, holiday/weekend indicators, previous $24 \mathrm{~h}$ mean electricity demand, $24 \mathrm{~h}$ lagged electricity demand, and $168 \mathrm{~h}$ lagged electricity demand. While the target variable is the electricity demand of the microgrid.

The magnitudes of WT extracted details of the electricity demand dataset and predictor dataset collected for one year (from 1 May 2014 to 30 April 2015) are employed to train the FFANN model using the SA parameter optimization method.

Different independent experimentation trials have been performed to determine the optimal configuration of the FFANN model and the training algorithm, SA. The best configuration was selected using the root mean squared error (RMSE) measure between the model output value and actual target value. Twelve independent experimentation trials were carried out and the one which achieved the lowest RMSE was selected to form the final prediction model parameters. Hence, the results depicted in this paper are based on the best SA and FFANN parameters obtained from the parameterization experiment which achieved the lowest RMSE.

The prediction performance of the devised forecast model is verified with a one year (from May 2015 to April 2016) testing window length. However, to illustrate the testing results here, the model testing result is given for randomly chosen four weekdays and four weekends/holidays representing the weekdays and weekends of the four seasons of the year: summer weekday (Wednesday-22 July 2015), summer weekend (Sunday -26 July 2015), fall weekday (Thursday-15 October 2015), fall weekend (Saturday-17 October 2015), winter weekday (Monday-4 January 2016), winter holiday (Friday-1 
January 2016), spring weekday (Wednesday-13 April 2016), and spring weekend (Sunday—24 April 2016). Hence, particular days with better electricity demand profiles are not chosen deliberately. This demonstrates an irregular forecast accuracy distribution over the testing year that reveals the actual power consumption in the microgrid system.

The forecast results are presented for the testing days with one-hour resolution. The electricity demand forecasts by the proposed integrated WT-SA-FFANN AI method are depicted in Figures 11 and 12, for winter, spring, summer and fall weekdays and weekends, respectively. The figures show the real electricity demand versus the forecasted demand by the devised method.
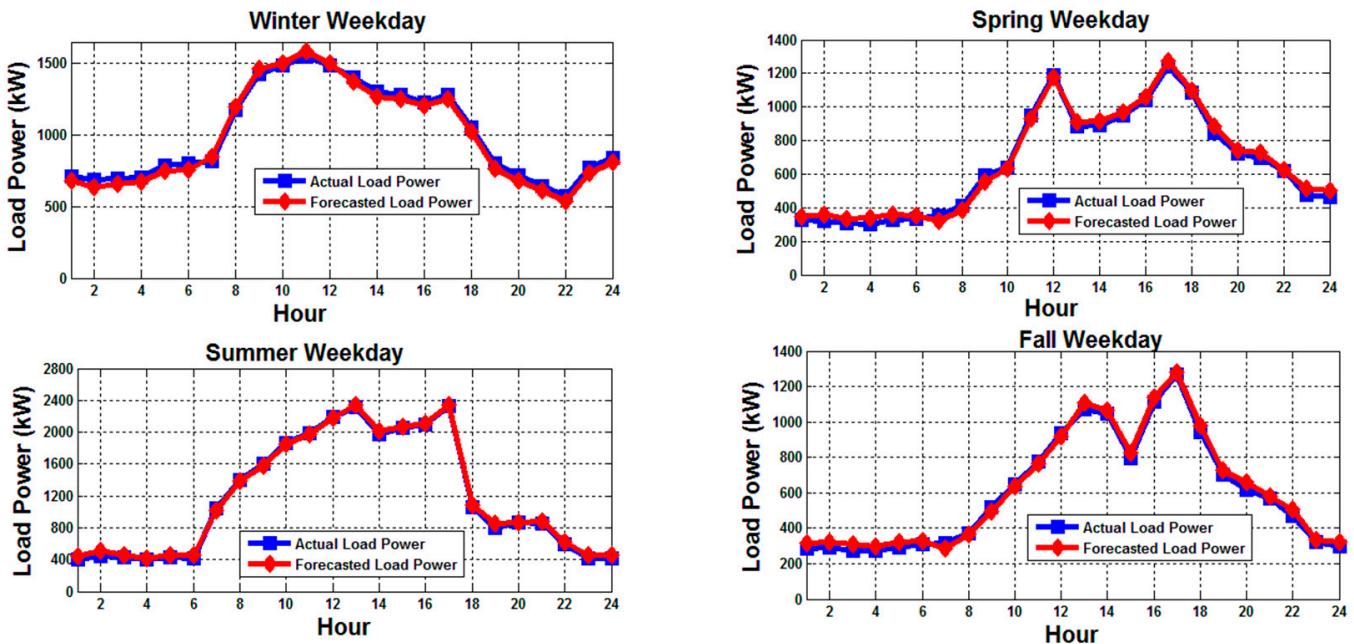

Figure 11. Real vs. forecasted electricity demand for weekdays.
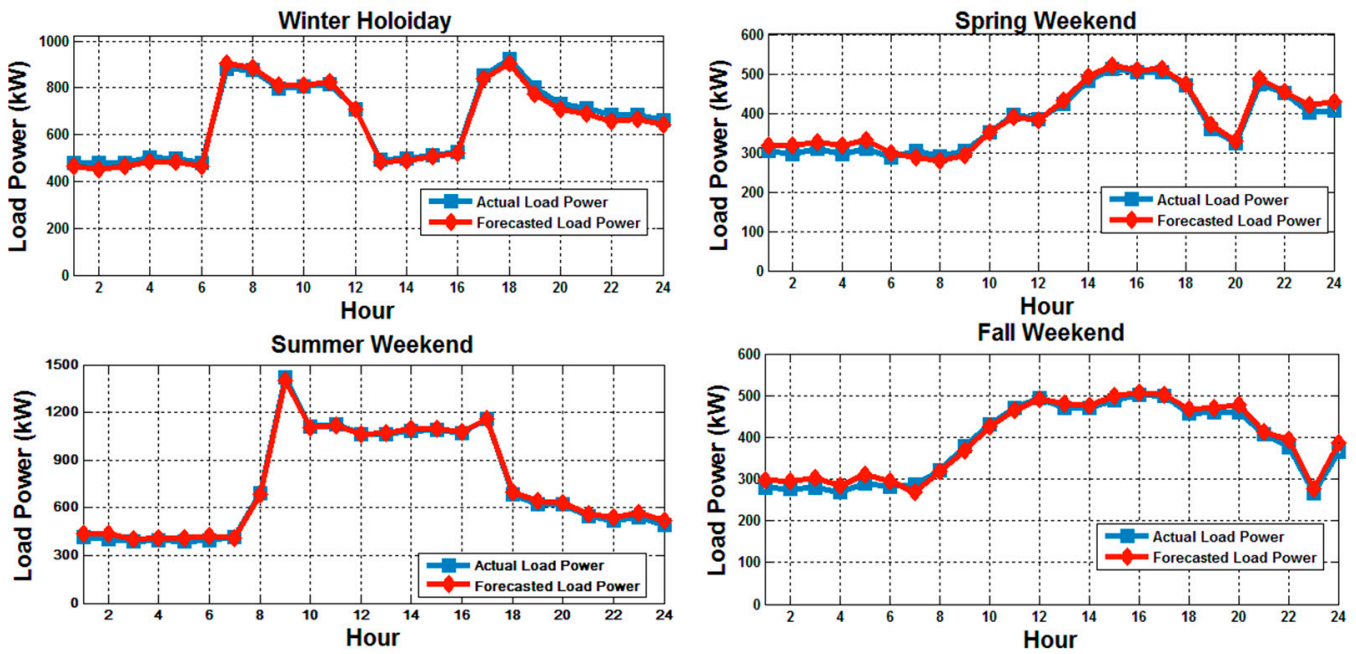

Figure 12. Real vs. forecasted electricity demand for weekends/holidays.

As it can be observed in Figures 11 and 12, the forecasts by the devised integrated WT-SA-FFANN AI-based approach follow the actual load power trends with smaller gaps (errors) between them. However, as we compare the performance of the devised approach between the weekdays and weekends, it is seen that the prediction accuracy is lower during the weekdays. This is because there might be some uncertain conditions in weekdays (working days) which could affect the electricity consumptions in the microgrid, such as for example the number of people coming or leaving the industrial company in weekdays. On the contrary, there are less uncertainties in weekends (non-working days) since several electrical loads are generally switched off and lesser fluctuation of the power-plugged loads, and there would be less number of electricity consumers (employees do not come to work and use 
electricity) in weekends. Thus, these facts result in less uncertainty (error) or higher accuracy of forecasts in weekends compared to weekdays.

Besides, as we compare the performance of the devised AI-based integrated approach among the seasons, it is observed that the forecast errors are higher during winter and summer seasons. This is because there are higher consumptions of electricity and much uncertainty in winter and summer seasons due to environmental (weather) impacts. There are more electricity consumptions for winter heating and summer cooling purposes, for reacting the weather discomforts in these seasons. Thus, the increased electricity demand combined with the higher weather fluctuation in the winter and summer seasons caused lower forecast accuracy compared to the spring and fall seasons in which both the electricity demands and weather conditions are relatively less fluctuating.

Table 3 provides the values of the criteria employed to estimate the forecasting error of the proposed integrated WT-SA-FFANN AI-based approach for day-ahead electricity demand forecasting in microgrids.

Table 3. Forecasting performance analysis of the proposed model (WT-SA-FFANN).

\begin{tabular}{ccccccc}
\hline \multicolumn{2}{c}{ Day Type } & MAPE (\%) & RMSE (kW) & NMAE (\%) & SDE (kW) & Forecast Skill (\%) \\
\hline \multirow{2}{*}{ Winter } & Weekday & 3.30 & 34.44 & 1.43 & 32.17 & 49.15 \\
& Holiday & 2.37 & 17.28 & 0.67 & 15.11 & 62.86 \\
\hline \multirow{2}{*}{ Spring } & Weekday & 3.80 & 27.27 & 1.09 & 24.72 & 59.90 \\
& Weekend & 2.98 & 13.09 & 0.49 & 11.9 & 69.84 \\
\hline \multirow{2}{*}{ Summer } & Weekday & 2.24 & 29.37 & 1.14 & 28.41 & 57.18 \\
& Weekend & 1.84 & 16.26 & 0.58 & 14.61 & 67.76 \\
\hline \multirow{2}{*}{ Fall } & Weekday & 4.00 & 25.89 & 1.04 & 22.94 & 62.40 \\
& Weekend & 3.13 & 13.66 & 0.52 & 12.38 & 72.74 \\
\hline \multirow{2}{*}{ Average } & Weekday & 3.33 & 29.24 & 1.17 & 27.06 & 57.16 \\
& Weekend & 2.58 & 15.07 & 0.57 & 13.5 & 68.30 \\
\hline \multirow{2}{*}{ Total Average } & & $\mathbf{2 . 9 5}$ & $\mathbf{2 2 . 1 5}$ & $\mathbf{0 . 8 7}$ & $\mathbf{2 0 . 2 8}$ & $\mathbf{6 2 . 7 3}$ \\
\hline
\end{tabular}

Table 4 presents an effectiveness comparison between the devised integrated WT-SA-FFANN AI-based electricity demand forecasting strategy and other four strategies (Persistence, BP-FFANN, GA-FFANN, and PSO-FFANN), based on the MAPE criteria. The devised integrated strategy provides reduced forecast error; the total mean MAPE obtained is $2.95 \%$. The devised strategy's total mean MAPE increment compared to the other four strategies is $63.62 \%, 51.64 \%, 45.37 \%$ and $39.67 \%$, respectively. The same training dataset is used for each of the presented approaches and each approach is implemented with its optimal parameters and setups.

Table 4. Comparative MAPE (\%).

\begin{tabular}{clccccc}
\hline \multirow{2}{*}{ Winter } & & Persistence & BP-FFANN & GA-FFANN & PSO-FFANN & WT-SA-FFANN \\
& Weekday & 6.53 & 5.00 & 4.43 & 4.14 & 3.30 \\
& Holiday & 6.47 & 4.67 & 3.90 & 3.42 & 2.37 \\
\hline \multirow{2}{*}{ Spring } & Weekday & 9.99 & 7.66 & 6.82 & 6.30 & 3.80 \\
& Weekend & 9.52 & 7.17 & 6.43 & 5.56 & 2.98 \\
\hline \multirow{2}{*}{ Summer } & Weekday & 5.59 & 4.25 & 3.74 & 3.51 & 2.24 \\
& Weekend & 5.18 & 4.04 & 3.68 & 3.24 & 1.84 \\
\hline \multirow{2}{*}{ Fall } & Weekday & 11.00 & 8.17 & 7.24 & 6.80 & 4.00 \\
& Weekend & 10.85 & 7.87 & 6.98 & 6.15 & 3.13 \\
\hline \multirow{2}{*}{ Average } & Weekday & 8.23 & 6.27 & 5.56 & 5.19 & 3.33 \\
& Weekend & 8.00 & 5.94 & 5.25 & 4.59 & 2.58 \\
\hline \multirow{2}{*}{ Total Average } & & $\mathbf{8 . 1 1}$ & $\mathbf{6 . 1 0}$ & $\mathbf{5 . 4 0}$ & $\mathbf{4 . 8 9}$ & $\mathbf{2 . 9 5}$ \\
\hline
\end{tabular}


Table 5 presents the electricity demand prediction accuracy assessment regarding the NMAE criterion, taking into account the devised integrated WT-SA-FFANN strategy and the other four strategies (Persistence, BP-FFANN, GA-FFANN, and PSO-FFANN).

Table 5. Comparative NMAE (\%).

\begin{tabular}{clccccc}
\hline \multirow{2}{*}{ Winter } & & Persistence & BP-FFANN & GA-FFANN & PSO-FFANN & WT-SA-FFANN \\
& Weekday & 2.837 & 2.163 & 1.915 & 1.791 & 1.426 \\
& Holiday & 1.839 & 1.327 & 1.109 & 0.973 & 0.675 \\
\hline \multirow{2}{*}{ Spring } & Weekday & 2.852 & 2.188 & 1.947 & 1.798 & 1.086 \\
& Weekend & 1.723 & 1.256 & 1.053 & 0.909 & 0.489 \\
\hline \multirow{2}{*}{ Summer } & Weekday & 2.843 & 2.162 & 1.904 & 1.786 & 1.140 \\
& Weekend & 1.961 & 1.410 & 1.168 & 1.027 & 0.583 \\
\hline \multirow{2}{*}{ Fall } & Weekday & 2.855 & 2.121 & 1.881 & 1.767 & 1.038 \\
& Weekend & 1.967 & 1.391 & 1.159 & 1.020 & 0.519 \\
\hline \multirow{2}{*}{ Average } & Weekday & 2.844 & 2.159 & 1.912 & 1.785 & 1.172 \\
& Weekend & 1.872 & 1.346 & 1.122 & 0.982 & 0.566 \\
\hline \multirow{2}{*}{ Total Average } & & $\mathbf{2 . 3 5 8}$ & $\mathbf{1 . 7 5 2}$ & $\mathbf{1 . 5 1 7}$ & $\mathbf{1 . 3 8 3}$ & $\mathbf{0 . 8 6 9}$ \\
\hline
\end{tabular}

Concerning the NMAE criterion as provided in Table 5, the devised WT-SA-FFANN integrated approach gives a total (all day types) average error representing $0.87 \%$ of the peak aggregate electricity demand of the microgrid for its day-ahead predictions across the entire forecast horizon.

The electricity demand prediction hourly normalized absolute error (NAE) (normalized by the maximum electricity demand), for all the considered models, are depicted in Figures 13 and 14, respectively, for weekdays and weekends. It is shown in the figures that the devised WT-SA-FFANN based electricity demand forecasting integrated approach provides the lowest NAEs compared to the other four evaluated approaches. Moreover, the normalized absolute errors during weekends/holidays (non-working days) are lower than that of weekdays (working days). Also, the normalized absolute errors for winter and summer days are relatively lower compared to spring and fall days.

Besides the MAPE and NMAE criteria, regularity of forecasts is another key factor for comparing the performances of electricity demand prediction models. Table 6 provides the performance comparison between the devised integrated WT-SA-FFANN based electricity demand prediction strategy and the other four strategies (Persistence, BP-FFANN, GA-FFANN, and PSO-FFANN), based on the daily forecast error variance. 

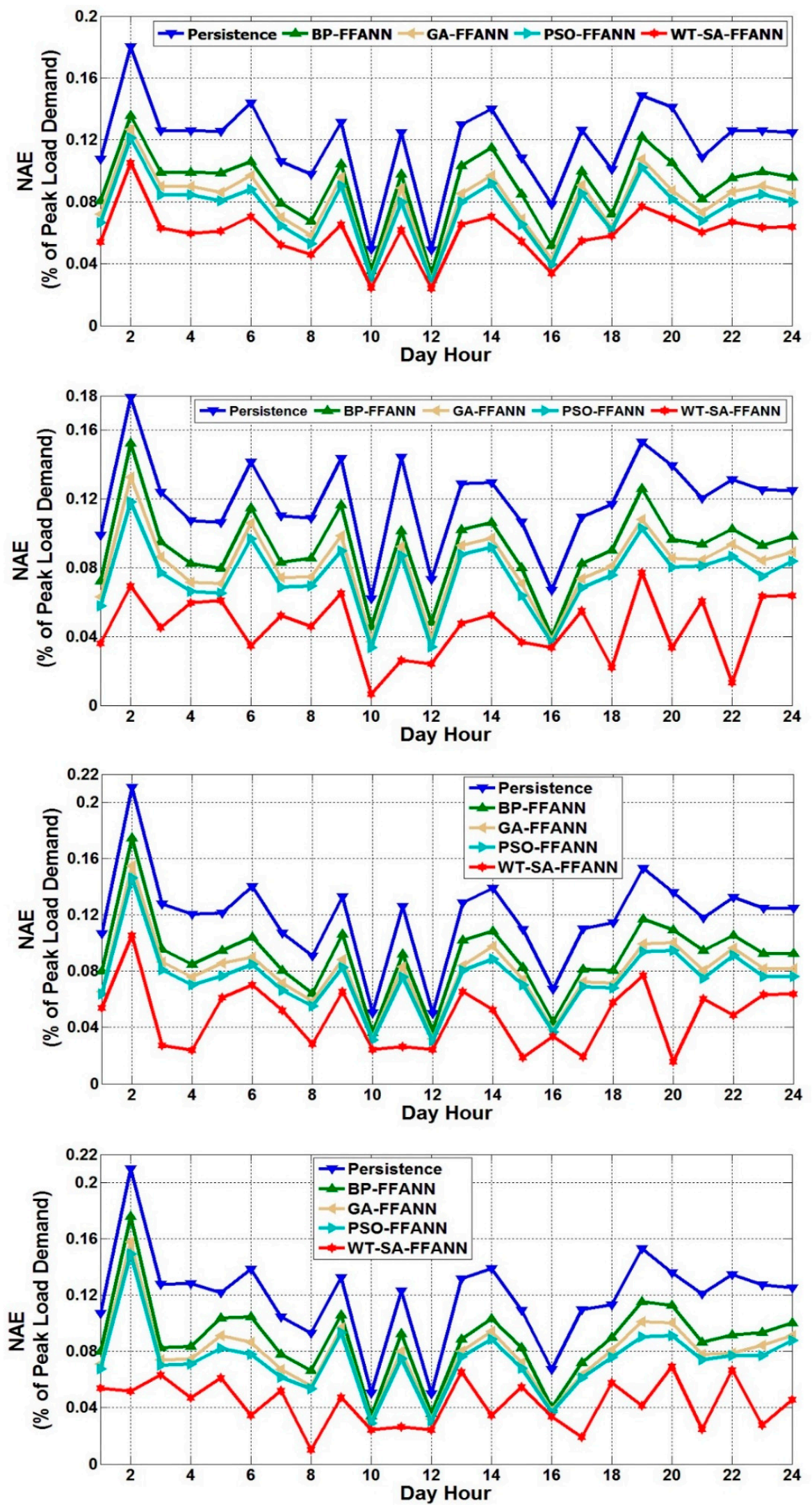

Figure 13. NAE for weekdays (the first curve for the winter weekday, second curve for the spring weekday, third curve for the spring weekday and fourth curve for the fall weekday). 

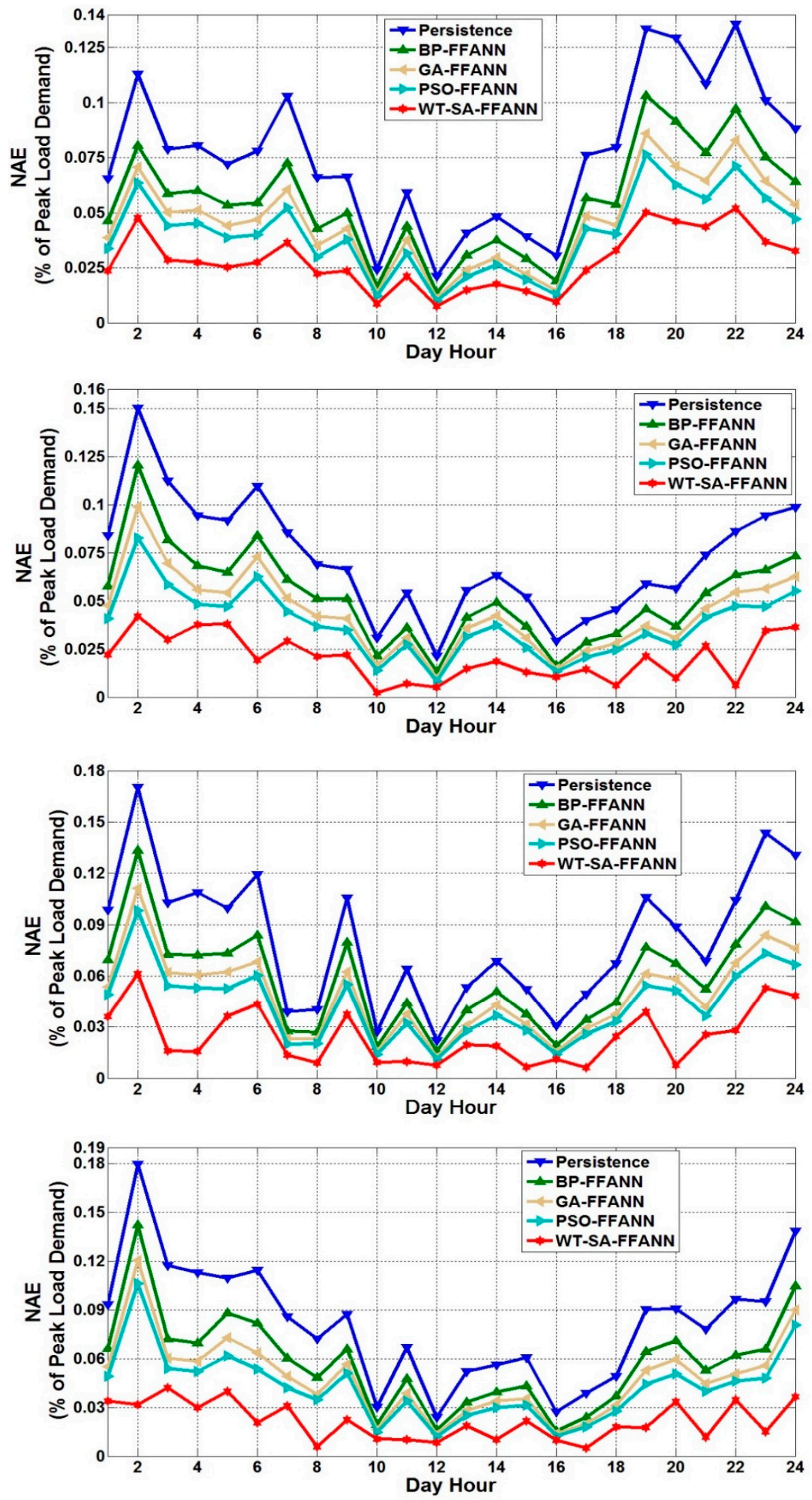

Figure 14. NAE for weekends/holidays (the first curve for the winter holiday, second curve for the spring weekend, third curve for the spring weekend and fourth curve for the fall weekend). 
Table 6. Comparative forecast error-variance.

\begin{tabular}{clccccc}
\hline \multirow{2}{*}{ Winter } & & Persistence & BP-FFANN & GA-FFANN & PSO-FFANN & WT-SA-FFANN \\
\hline \multirow{2}{*}{ Spring } & Weekday & 0.00025 & 0.00018 & 0.00015 & 0.00014 & 0.00008 \\
& Holiday & 0.00020 & 0.00017 & 0.00014 & 0.00013 & 0.00007 \\
\hline \multirow{2}{*}{ Summer } & Weekday & 0.00049 & 0.00042 & 0.00033 & 0.00029 & 0.00021 \\
& Weekend & 0.00045 & 0.00041 & 0.00032 & 0.00028 & 0.00020 \\
\hline \multirow{2}{*}{ Fall } & Weekday & 0.00023 & 0.00017 & 0.00013 & 0.00012 & 0.00010 \\
& Weekend & 0.00020 & 0.00016 & 0.00012 & 0.00011 & 0.00008 \\
\hline \multirow{2}{*}{ Average } & Weekday & 0.00088 & 0.00068 & 0.00055 & 0.00047 & 0.00024 \\
& Weekend & 0.00058 & 0.00054 & 0.00051 & 0.00043 & 0.00021 \\
\hline \multirow{2}{*}{ Total Average } & Weekday & 0.00046 & 0.00036 & 0.00029 & 0.00025 & 0.00016 \\
\hline
\end{tabular}

As Table 6 presented, the mean error variance achieved by the devised integrated approach is smaller, indicating lower uncertainty of the electricity demand predictions. The devised model's total mean error variance increment compared to the other four models is $63.41 \%, 57.14 \%, 46.43 \%$, and $37.5 \%$, respectively.

On top of the accuracy evaluation criteria presented above, the forecast skill FS can also be an important performance index for evaluating various electricity demand prediction approaches. It indicates the quality of a prediction approach by referring the approach's performance to persistence prediction. Table 7 provides an effectiveness contrast between the devised integrated WT-SA-FFANN electricity demand forecasting strategy and the other four strategies (Persistence, BP-FFANN, GA-FFANN, and PSO-FFANN), based on the FS criterion.

Table 7. Comparative Forecast Skill (\%).

\begin{tabular}{clccccc}
\hline \multirow{2}{*}{ Winter } & & Persistence & BP-FFANN & GA-FFANN & PSO-FFANN & WT-SA-FFANN \\
& Weekday & 0.0 & 23.00 & 31.67 & 36.00 & 49.15 \\
& Holiday & 0.0 & 27.57 & 39.31 & 46.74 & 62.86 \\
\hline \multirow{2}{*}{ Spring } & Weekday & 0.0 & 22.46 & 30.98 & 36.23 & 59.90 \\
& Weekend & 0.0 & 26.07 & 37.97 & 46.65 & 69.84 \\
\hline \multirow{2}{*}{ Summer } & Weekday & 0.0 & 23.22 & 32.35 & 36.57 & 57.18 \\
& Weekend & 0.0 & 27.48 & 39.88 & 47.15 & 67.76 \\
\hline \multirow{2}{*}{ Fall } & Weekday & 0.0 & 24.68 & 33.14 & 37.28 & 62.40 \\
& Weekend & 0.0 & 27.99 & 39.86 & 47.10 & 72.74 \\
\hline \multirow{2}{*}{ Average } & Weekday & 0.0 & 23.34 & 32.04 & 36.52 & 57.16 \\
& Weekend & 0.0 & 27.28 & 39.26 & 46.91 & 68.30 \\
\hline \multirow{2}{*}{ Total Average } & & $\mathbf{0 . 0}$ & $\mathbf{2 5 . 3 1}$ & $\mathbf{3 5 . 6 4}$ & $\mathbf{4 1 . 7 1}$ & $\mathbf{6 2 . 7 3}$ \\
\hline
\end{tabular}

As shown in Table 7, the devised integrated WT-SA-FFANN based electricity demand prediction approach gives the best forecast skill for all the testing days of all the seasons, magnifying its higher capability for electricity demand forecasting in microgrids.

\section{Conclusions}

In this study, a new and effective integrated technique is developed for day-ahead electricity demand forecasting in microgrids, considering electricity demand measurement records, weather forecasts, hours in the days, days in the weeks, and holiday/weekend indicators. The proposed method is based on the hybridization of the WT, SA and FFANN. In the devised approach, the fundamental prediction engine employed is the FFANN. The WT was used to decompose the initial target (electricity demand) dataset to a cluster of well-behaved subseries called details. The SA is utilized to optimize 
the FFANN weight parameters to obtain improved prediction accuracy. A one year (from May 2014 to April 2015) training window length with historical weather data (air temperature and humidity), microgrid electricity demand data and other supplementary data (hours in the days, days in the weeks and holiday/weekend indicators) are utilized to develop the forecast model of the devised strategy. The prediction accuracy of the devised strategy is tested with a one year (from May 2015 to April 2016) testing window length. The mean MAPE, NMAE and FS, for the tested days, are $2.95 \%, 0.87 \%$ and $62.73 \%$, respectively. The mean forecast execution time is lower than $15 \mathrm{~s}$ using MATLAB/Simulink (R2018b version). Thus, the numerical results and comparisons with other methods confirm the effectiveness of the proposed hybrid AI-based method for a day-ahead (short-term) electric load prediction in microgrids. The obtained microgrid electricity demand forecast results can be used as input information for the energy management system, demand response control, scheduling center, dispatching center, and other operational control units of the microgrid.

Author Contributions: Y.M. and M.Z. gathered the prediction model input dataset, examined the dataset, and proposed and implemented the prediction algorithm. All authors have read and approved the final manuscript.

Funding: This research was funded by the National Natural Science Foundation Project of China, 61473094.

Acknowledgments: The School of Electronic Information Engineering of Guangdong University of Petrochemical Technology, China supported this work financially and technically.

Conflicts of Interest: The authors declare no conflict of interest.

\section{References}

1. International Smart Grid Action Network (ISGAN). Energy Systems and Integration. Available online: https://www.iea-isgan.org/ (accessed on 3 February 2019).

2. Hernandez, L.; Baladrón, C.; Aguiar, J.M.; Carro, B.; Sanchez-Esguevillas, A.J.; Lloret, J. Short-term load forecasting for microgrids based on ANNs. Energies 2013, 6, 1385-1408. [CrossRef]

3. Hernández, L.; Baladrón, C.; Aguiar, J.M.; Calavia, L.; Carro, B.; Sánchez-Esguevillas, A.; Pérez, F.; Fernández, Á.; Lloret, J. Artificial Neural Network for Short-Term Load Forecasting in Distribution Systems. Energies 2014, 7, 1576-1598. [CrossRef]

4. Eseye, A.T.; Zhang, J.; Zheng, D.; Wei, D. Optimal energy management strategy for an isolated industrial microgrid using a modified PSO technique. In Proceedings of the 2016 IEEE International Conference on Power and Renewable Energy (ICPRE), Shanghai, China, 21-23 October 2016.

5. Eseye, A.T.; Zheng, D.; Li, H.; Zhang, J. Grid-price dependent optimal energy storage management strategy for grid-connected industrial microgrids. In Proceedings of the IEEE Green Tech Conference, Denver, CO, USA, 29-31 March 2017.

6. Mohammadi, S.; Keivani, H.; Bakhshi, M.; Mohammadi, A.; Kavehnia, M.R.F. Demand forecasting using time series modelling and ANFIS estimator. In Proceedings of the 41st IEEE International Universities Power Engineering Conference, Newcastle-upon-Tyne, UK, 6-8 September 2006.

7. Zheng, H.; Yuan, J.; Chen, L. Short-Term Load Forecasting Using EMD-LSTM neural networks with a Xgboost algorithm for feature importance evaluation. Energies 2017, 10, 1168. [CrossRef]

8. Xu, L.; Li, C.; Xie, X.; Zhang, G. Long-short-term memory network based hybrid model for short-term electrical load forecasting. Information 2018, 9, 165. [CrossRef]

9. Lee, W.; Hong, J. A hybrid dynamic and fuzzy time series model for mid-term power load forecasting. Int. J. Electr. Power Energy Syst. 2015, 64, 1057-1062. [CrossRef]

10. Zhang, X.; Wang, J. A novel decomposition-ensemble model for forecasting short-term load-time series with multiple seasonal patterns. Appl. Soft Comput. 2018, 65, 478-494. [CrossRef]

11. Ghanbari, S.; Ghaderi, F.; Azadeh, M.A. Adaptive neuro-fuzzy inference system vs. regression based approaches for annual electricity load forecasting. In Proceedings of the 2nd International Conference on Computer and Automation Engineering (ICCAE), Singapore, 26-28 February 2010.

12. Hernández, L.; Baladrón, C.; Aguiar, J.M.; Calavia, L.; Carro, B.; Sánchez-Esguevillas, A.; Sanjuán, J.; González, Á.; Lloret, J. Improved short-term load forecasting based on two-stage predictions with artificial neural networks in a microgrid environment. Energies 2013, 6, 4489-4507. [CrossRef] 
13. Ryu, S.; Noh, J.; Kim, H. Deep neural network based demand side short term load forecasting. Energies 2017, 10, 3. [CrossRef]

14. Chen, J.-F.; Do, Q.H.; Nguyen, T.V.A.; Doan, T.T.H. Forecasting monthly electricity demands by wavelet neuro-fuzzy system optimized by heuristic algorithms. Information 2018, 9, 51. [CrossRef]

15. Ruiz-Abellón, M.C.; Gabaldón, A.; Guillamón, A. Load forecasting for a campus university using ensemble methods based on regression trees. Energies 2018, 11, 2038. [CrossRef]

16. Sigauke, C.; Nemukula, M.M.; Maposa, D. Probabilistic hourly load forecasting using additive quantile regression models. Energies 2018, 11, 2208. [CrossRef]

17. Al-Kandari, A.M.; Soliman, S.A.; El-Hawary, M.E. Fuzzy short-term electric load forecasting. Int. J. Electr. Power Energy Systst. 2004, 26, 111-122. [CrossRef]

18. Mamlook, R.; Badran, O.; Abdulhadi, E. A fuzzy inference model for short-term load forecasting. Energy Policy 2009, 37, 1239-1248. [CrossRef]

19. Hassan, S.; Khosravi, A.; Jaafar, J.; Khanesar, M.A. A systematic design of interval type-2 fuzzy logic system using extreme learning machine for electricity load demand forecasting. Int. J. Electr. Power Energy Systst. 2016, 82, 1-10. [CrossRef]

20. Raza, M.Q.; Khosravi, A. A review on artificial intelligence based load demand forecasting techniques for smart grid and buildings. Renew. Sustain. Energy Rev. 2015, 50, 1352-1372. [CrossRef]

21. Mukhopadhyay, P.; Mitra, G.; Banerjee, S.; Mukherjee, G. Electricity load forecasting using fuzzy logic: Short term load forecasting factoring weather parameter. In Proceedings of the 2017 7th International Conference on Power Systems (ICPS), Pune, India, 21-23 December 2017.

22. Ghanbarian, M.; Kavehnia, F.; Askari, R.M.; Mohammadi, A.; Keivani, H. Applying time-series regression to load forecasting using neuro-fuzzy techniques. In Proceedings of the 2007 International Conference on Power Engineering, Energy and Electrical Drives, Setubal, Portugal, 12-14 April 2007.

23. Mourad, M.; Bouzid, B.; Mohamed, B. A hybrid wavelet transform and ANFIS model for short term electric load prediction. In Proceedings of the 2012 2nd International Conference on Advances in Computational Tools for Engineering Applications, Beirut, Lebanon, 12-15 December 2012.

24. Hayati, M.; Shirvany, Y. Artificial neural network approach for short term load forecasting for illam region. Int. J. Electr. Comput. Eng. 2007, 28, 280-284.

25. Hwang, H. Daily Electric load forecasting based on rbf neural network models. Int. J. Fuzzy Log. Intell. Systst. 2013, 13, 39-49. [CrossRef]

26. Senabre, C.; Valero, S.; Aparicio, J. Using a self organizing map neural network for short-term load forecasting, analysis of different input data patterns. In Distributed Computing and Artificial Intelligence. Advances in Intelligent and Soft Computing; Springer: Berlin/Heidelberg, Germany, 2010.

27. Deoras, A. Electricity Load and Price Forecasting with MATLAB. Mathwork's Webinar Case Study. Available online: https://www.mathworks.com/matlabcentral/fileexchange/28684-electricity-load-and-priceforecasting-webinar-case-study) (accessed on 1 October 2018).

28. Dolara, A.; Leva, S.; Mussetta, M.; Ogliari, E. PV hourly day-ahead power forecasting in a micro grid context. In Proceedings of the 2016 IEEE 16th International Conference on Environment and Electrical Engineering (EEEIC), Florence, Italy, 7-10 June 2016.

29. Dolara, A.; Grimaccia, F.; Leva, S.; Mussetta, M.; Ogliari, E. Comparison of Training Approaches for Photovoltaic Forecasts by Means of Machine Learning. Appl. Sci. 2018, 8, 228. [CrossRef]

30. Ogliari, E.; Niccolai, A.; Leva, S.; Zich, R.E. Computational intelligence techniques applied to the day ahead PV output power forecast: PHANN, SNO and mixed. Energies 2018, 11, 1487. [CrossRef]

31. Eseye, A.T.; Zhang, J.; Zheng, D. Short-term photovoltaic solar power forecasting using a hybrid wavelet-PSO-SVM model based on SCADA and meteorological information. Renew. Energy 2018, 118, 357-367. [CrossRef]

32. Zheng, D.; Shi, M.; Wang, Y.; Eseye, A.T.; Zhang, J. Day-ahead wind power forecasting using a two-stage hybrid modeling approach based on SCADA and meteorological information and evaluating the impact of input-data dependency on forecasting accuracy. Energies 2017, 10, 1988. [CrossRef]

33. Coimbra, C.; Kleissl, J.; Marquez, R. Overview of solar forecasting methods and a metric for accuracy evaluation. In Solar Resource Assessment and Forecasting; Kleissl, J., Ed.; Elsevier: Waltham, MA, USA, 2013.

34. Timeanddate.com. Calendar for Year 2014, 2015 and 2016 (China). Available online: https://www.timeanddate. com/calendar/ (accessed on 6 October 2018). 
35. Amjady, N.; Keynia, F. Short-term load forecasting of power systems by combination of wavelet transform and neuro-evolutionary algorithm. Energy 2009, 34, 46-57. [CrossRef]

36. Reis, J.R.; da Silva, A.P.A. Feature extraction via multiresolution analysis for short-term load forecasting. IEEE Trans. Power Syst. 2005, 20, 189-198.

37. Mallat, S. A theory for multiresolution signal decomposition-The wavelet representation. IEEE Trans. Pattern Anal. Mach. Intell. 1989, 11, 674-693. [CrossRef]

38. Amjady, N.; Keynia, F. Day ahead price forecasting of electricity markets by a mixed data model and hybrid forecast method. Int. J. Electr. Power Energy Syst. 2008, 30, 533-546. [CrossRef]

39. Khachaturyan, A.; Semenovskaya, S.; Vainshtein, B. Statistical-Thermodynamic Approach to Determination of Structure Amplitude Phases. Sov. Phys. Crystallogr. 1979, 24, 519-524.

40. Khachaturyan, A.; Semenovskaya, S.; Vainshtein, B. The thermodynamic approach to the structure analysis of crystals. Acta Crystallogr. 1981, 37, 742-754. [CrossRef]

41. Metropolis, N.; Rosenbluth, A.; Rosenbluth, M.; Teller, A.; Teller, E. Equation of state calculations by fast computing machines. J. Chem. Phys. 1953, 21, 1087-1090. [CrossRef]

42. Kirkpatrick, S.; Gelatt, C.D., Jr.; Vecchi, M.P. Optimization by simulated annealing. Science 1983, 220, 671-680. [CrossRef]

(C) 2019 by the authors. Licensee MDPI, Basel, Switzerland. This article is an open access article distributed under the terms and conditions of the Creative Commons Attribution (CC BY) license (http://creativecommons.org/licenses/by/4.0/). 